\title{
Detailed radio to soft $\gamma$-ray studies of the 2005 outburst of the new X-ray transient XTE J1818-245
}

\author{
M. Cadolle Bel ${ }^{1}$, L. Prat ${ }^{2,3}$, J. Rodriguez ${ }^{2,3}$, M. Ribó ${ }^{4}$, L. Barragán ${ }^{1,5,6}$, P. D’Avanzo ${ }^{7,8}$, D. C. Hannikainen ${ }^{9}$, \\ E. Kuulkers ${ }^{1}$, S. Campana ${ }^{7,8}$, J. Moldón ${ }^{4}$, S. Chaty ${ }^{2,3}$, J. Zurita-Heras ${ }^{2,3}$, A. Goldwurm ${ }^{2,10}$, and P. Goldoni ${ }^{2,10}$ \\ 1 ESAC, ISOC, Villañueva de la Cañada, Madrid, Spain \\ e-mail: Marion. Cadolle@sciops.esa.int \\ 2 CEA-Saclay, DSM/IRFU/SAp, France \\ AIM UMR 7158, Paris, France \\ ${ }^{4}$ Departament d'Astronomia i Meteorologia and Institut de Ciències del Cosmos (ICC), Universitat de Barcelona (UB/IEEC), \\ Martí i Franquès 1, 08028 Barcelona, Spain \\ 5 Dr. Karl Remeis Sternwarte, Sternwartstr. 7, 96049 Bamberg, Germany \\ ${ }_{7}^{6}$ ECAP, Erwin-Rommel-Straße 1, 91058 Erlangen, Germany \\ 7 INAF, Osservatorio Astronomico di Brera, Merate, Italy \\ 8 Università degli Studi dell'Insubria, Como, Italy \\ 9 Metsähovi Radio Observatory, Helsinki University of Technology TKK, Metsähovintie 114, 02540 Kylmälä, Finland \\ 10 APC-UMR 7164, Paris, France
}

Received 4 August 2008 / Accepted 18 March 2009

\begin{abstract}
Context. XTE J1818-245 is an X-ray nova that experienced an outburst in 2005, as first seen by the RXTE satellite. The source was observed simultaneously at various wavelengths up to soft $\gamma$-rays with the INTEGRAL satellite, from 2005 February to September, during our INTEGRAL Target of Opportunity program dedicated to new X-ray novae and during Galactic Bulge observations.

Aims. X-ray novae are extreme systems that often harbor a black hole, and are known to emit throughout the electromagnetic spectrum when in outburst. The goals of our programme are to understand the physical processes close to the black hole and to study the possible connection with the jets that are observed in the radio.

Methods. We analysed radio, (N)IR, optical, X-ray and soft $\gamma$-ray observations. We constructed simultaneous broad-band X-ray spectra covering a major part of the outburst, which we fitted with physical models. Analyzing both the light curves in various energy ranges and the hardness-intensity diagram enabled us to study the long-term behaviour of the source.

Results. Spectral parameters were typical of the soft intermediate states and the high soft states of a black hole candidate. The source showed relatively small spectral variations in X-rays with considerable flux variation in radio. Spectral studies showed that the accretion disc cooled down from 0.64 to $0.27 \mathrm{keV}$ in $\sim 100$ days and that the total flux decreased while the relative flux of the hot medium increased. Radio emission was detected several times, and, interestingly, five days after entering the HSS. Modeling the spectral energy distribution from the radio to the soft $\gamma$-rays reveals that the radio flares arise from several ejection events.

Conclusions. XTE J1818-245 probably belongs to the class of low-mass X-ray binaries and is likely a black hole candidate transient source that might be closer than the Galactic Bulge. The results from the data analysis trace the physical changes that took place in the system (disc, jet/corona) at a maximum bolometric luminosity of $0.4-0.9 \times 10^{38} \mathrm{erg} \mathrm{s}^{-1}$ (assuming a distance between $2.8-4.3 \mathrm{kpc}$ ) and they are discussed within the context of disc and jet models.
\end{abstract}

Key words. black hole physics - stars: individual: XTE J1818-245 - gamma rays: observations - X-rays: binaries - infrared: general - radio continuum: general

\section{Introduction}

$\mathrm{X}$-ray novae $(\mathrm{XNe})$, also known as soft X-ray transients, are accreting low-mass X-ray binaries (LMXB) that spend most of their time in a faint, quiescent state. They undergo large amplitude outbursts with rise times of only a few days or weeks, with typical recurrence periods of many years (Tanaka \& Shibazaki 1996). The picture commonly accepted for an XN involves the transition from a low-mass accretion rate state to a high-mass accretion rate, optically thick accretion flow, triggered by an accretion disc instability. The optically thick and geometrically thin accretion disc has a varying inner radius and temperature, emitting at typical X-ray energies of $\sim 1 \mathrm{keV}$. This region is probably surrounded by a hot corona, where soft X-ray photons originating in the disc undergo inverse Comptonization, emitting a power law spectrum up to $\gamma$-rays. A relativistic jet might be present, typically observed in the radio. These spectral characteristics are coupled to different levels of variability, to quasi periodic oscillations (QPOs) observed in the power spectrum spectrum (e.g., Belloni et al. 2001; Belloni 2005) and to changes in the radio. Depending on the relative strengths of each component and on how they vary, several spectral states have been identified: see, e.g., McClintock \& Remillard (2006), Homan \& Belloni (2005). In the second classification, the two main spectral states are the low/hard state (LHS), dominated by non-thermal emission and the high soft state (HSS), dominated 
Table 1. Log of the XTE J1818-245 observations analysed in this paper.

\begin{tabular}{|c|c|c|c|c|c|c|}
\hline Observatory & Instrument & Bandpass & $\begin{array}{c}\text { Period } \\
(\text { MJD-53596.5) })^{a}\end{array}$ & $\begin{array}{c}\text { Total Exposure } \\
(\mathrm{ks})\end{array}$ & $\begin{array}{l}\text { Number } \\
\text { of Obs. }\end{array}$ & Observation Type \\
\hline \multirow[t]{2}{*}{ INTEGRAL } & IBIS/ISGRI & $18-200 \mathrm{keV}$ & $2.5-23.8$ & 315 & $3 / 4$ & ToO $\left(5 \times 5^{b}\right) /$ Galactic Bulge $^{c}\left(\right.$ hex $\left.^{d}\right)$ \\
\hline & JEM-X & $5-25 \mathrm{keV}$ & $5.9-14.8$ & 240 & 3 & $\mathrm{ToO}$ \\
\hline \multirow[t]{2}{*}{$R X T E$} & PCA & $3-25 \mathrm{keV}$ & $3.1-103.3$ & 146 & 54 & Public \\
\hline & HEXTE & $20-150 \mathrm{keV}$ & $3.1-103.3$ & 146 & 54 & Public \\
\hline Swift ${ }^{e}$ & XRT & $0.3-10 \mathrm{keV}$ & 5.8 & 0.13 & 1 & Public \\
\hline $\mathrm{REM}^{e}$ & ROSS & $550-800 \mathrm{~nm}$ & $22.7-76.5$ & 3.2 & 10 & $\mathrm{ToO}$ \\
\hline $\mathrm{NTT}^{e}$ & SUSI-2 & $320-900 \mathrm{~nm}$ & $9.6-0.6$ & 1 & $\mathrm{ToO}$ & \\
\hline VLA $^{e}$ & $\mathrm{~L}, \mathrm{C}, \mathrm{X}, \mathrm{U}$ & $1.4-15 \mathrm{GHz}$ & $5.6-196.01$ & $4.8 / 15.4$ & $1 / 13$ & ToO/Public \\
\hline VLBA $^{e}$ & $\mathrm{~S} / \mathrm{X}$ & 2.3-8.4 GHz & $8.54-8.67$ & 10.8 & 1 & $\mathrm{ToO}$ \\
\hline
\end{tabular}

Notes:

${ }^{a}$ MJD 53596.5 corresponds to the maximum flux of the source measured during the period covered by RXTE/ASM.

$b 5 \times 5$ dither pattern around the nominal target location.

${ }^{c}$ Monitoring program of E. Kuulkers et al. (see Kuulkers et al. 2007).

${ }^{d}$ Hexagonal pattern around the nominal target location.

${ }^{e}$ Snap-shot observations with the specified instruments or receivers.

by emission from the accretion disc. In the LHS, the fast time variability is dominated by strong $(\sim 30 \%$ fractional $\mathrm{rms})$ bandlimited noise. At times, low-frequency QPOs are present. In this state, flat-spectrum radio emission is observed, associated with compact jet ejection (Corbel et al. 2000, 2003; Gallo et al. 2003, 2006; Fender et al. 2004). In the HSS, only weak power law noise is present in the power spectrum. No core radio emission is detected (see Fender et al. 1999, 2005: "quenching" of the jet). Further states have been identified as "intermediate" based on the above-mentioned differences in the soft/hard X-ray components, variability and radio emission: the HIMS (hard intermediate state) and SIMS (soft intermediate state). In the HIMS, the energy spectrum is softer than in the LHS, with evidence for a soft thermal disc component. The power spectra feature bandlimited noise with characteristic frequency higher than the LHS and usually a rather strong $0.1-15 \mathrm{~Hz}$ type-C QPO (see, e.g., Casella et al. 2005). In the SIMS, the disc component dominates the flux. No strong band-limited noise is observed but transient type-A and type-B QPOs are seen (the frequency of which spans only a limited range). As in the HSS, no core radio emission is detected.

XTE J1818-245 was discovered by the All-Sky Monitor (ASM) telescope on board the RXTE satellite on 2005 August 12. The ASM hardness ratio (hereafter, HR) indicated a very soft spectrum, often associated with a black hole $(\mathrm{BH})$ as the compact object of the binary system (Levine et al. 2005). Follow-up RXTE/Proportional Counter Array (PCA) observations provided a refined position and no pulsations were detected (Markwardt et al. 2005). On August 16-17, the INTEGRAL Soft Gamma-Ray Imager (IBIS/ISGRI) observed the source for 12.6 ks during the Galactic Bulge (GB) program: Shaw et al. (2005) found a position in the ISGRI mosaic image consistent with (and with smaller uncertainties) than the one found by the RXTE instruments. Soon after, the optical counterpart was identified (Steeghs et al. 2005) and the Swift/X-ray Telescope, XRT, (Still et al. 2005) improved the X-ray position. Finally, Rupen et al. (2005) detected XTE J1818-245 with the Very Large Array (VLA) at the optical position: the flux densities at $4.9 \mathrm{GHz}$ increased from $\sim 7$ to 27 mJy between August 20-21.

Based on the above-mentioned characteristics, XTE J1818-245 was suggested to be a BH candidate
(BHC). Target of Opportunity (ToO) observations for $\mathrm{XNe}$ in outburst were triggered with INTEGRAL, associated with a large multi-wavelength campaign. We report here the results of the INTEGRAL observations of XTE J1818-245 together with Swift, RXTE and NIR/optical/radio data. We start with a description of the available data and of the analysis procedures employed in Sect. 2. Results are presented in Sect. 3, followed by the interpretations and discussions in Sect. 4. We summarize our conclusions in Sect. 5.

\section{Observations and data reduction}

Table 1 summarizes the observations analysed in this paper, giving for each of them the instrument data available, energy ranges, dates, exposure times and modes. Figure 1 shows the $R X T E / A S M$ soft X-ray light curve of the outburst, with the time of the multiwavelength observations indicated.

\subsection{INTEGRAL}

Target of Opportunity observations were performed on (2005) August 20-21, 23 and 28-29, corresponding to revolutions (hereafter, Rev.) 348, 349 and 351, respectively. We also used the data from public observations performed during the GB monitoring program.

The IBIS/ISGRI and JEM-X data were reduced with the standard analysis procedures of the Off-Line Scientific Analysis OSA 7.0 released by ISDC, with algorithms described in Goldwurm et al. (2003) and Westergaard et al. (2003) for ISGRI and JEM-X respectively. Systematic errors of $2 \%$ were added for both JEM-X (in the 5-25 keV range) and ISGRI (in the 18-200 keV range). We used the maps, the response matrices and the off-axis and background corrections from OSA 7.0. For ISGRI, we rebinned the standard spectra to obtain between 4 and 10 spectral points, depending on the brightness of the source. We checked that the spectral index did not change by more than $2 \%$ during a single revolution (INTEGRAL orbit around the Earth lasting $\sim 3$ days): this allowed us to sum the flux per INTEGRAL Rev. to improve the signal-to-noise ratio for both light curves and spectra. The ISGRI image and light curves are respectively shown in Figs. 2 and 3 a. 


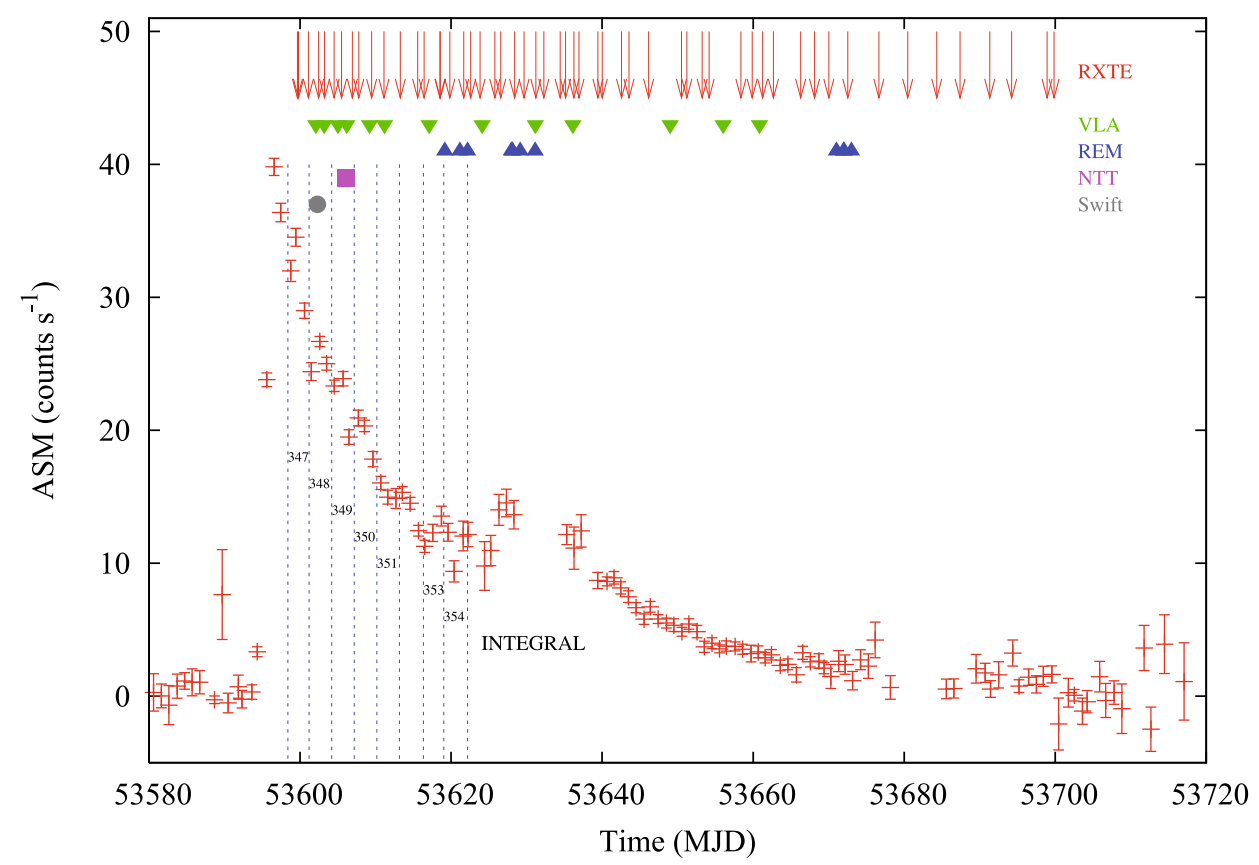

Fig. 1. RXTE/ASM light curve of XTE J1818-245 during the 2005 outburst. The times of the observations are indicated for each instrument as well as the INTEGRAL revolutions (lasting $\sim 3$ days between consecutive vertical dashed lines). Other observations lasting from 100 to $3000 \mathrm{~s}$ are indicated (see Table 1).

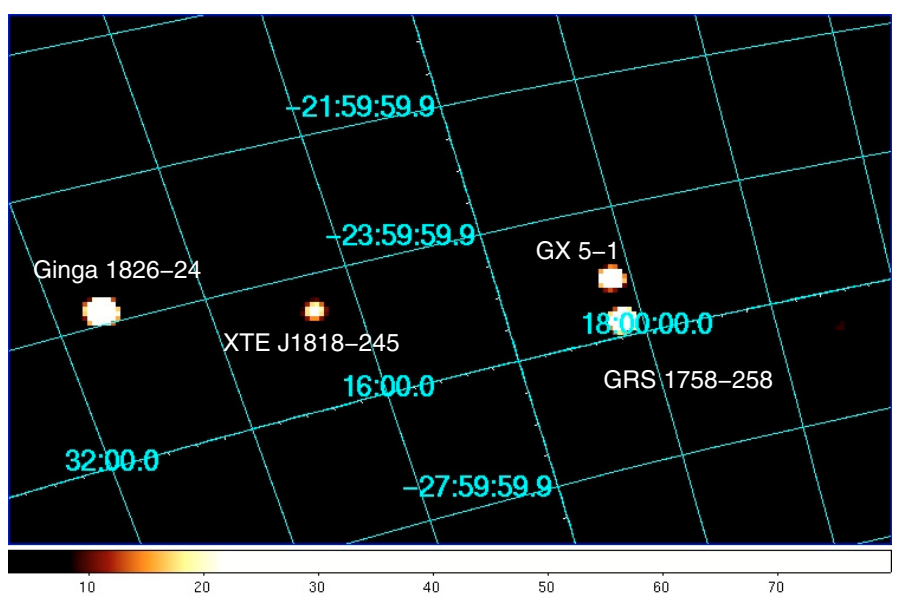

Fig. 2. INTEGRAL 20-40 keV IBIS/ISGRI reconstructed sky image (logarithmic black-body colour scale) of the region around XTE J1818-245 during our first ToO (Rev. 348, 70 ks exposure). The source appears at a significance level of $18 \sigma$ over the background, the type I X-ray burster Ginga 1826-24 at $129 \sigma$, the microquasar GRS $1758-258$ at $86 \sigma$ and the neutron star GX 5-1 at $65 \sigma$.

We could only perform JEM-X analysis on our ToO data as the source was outside the field of view (FOV) of JEM-X in the GB monitoring program. Since the RXTE/PCA instrument observes the source more frequently and has a higher sensitivity than JEM-X, the JEM-X data were not included in the broad-band spectra so as to be consistent over all our data sets. However, we verified that the best-fit spectral parameters using JEM-X and PCA were consistent within the error bars.

\subsection{RXTE}

We analysed all available observations, taken about once every two days from MJD 53599 to 53700 . Each observation lasted between 1 and $3.3 \mathrm{ks}$. The RXTE data were reduced with the
HEASOFT software package v6.4, following standard procedures (see Rodriguez et al. 2003). Energy spectra were only extracted from detector 2 of the PCA top layer.

We used the latest calibration files provided by the RXTE GOF (guest observer facility), which include important corrections applied to the PCA background since 2007 September 18. To determine the level of systematic errors, we used Crab spectra which were fitted with a model consisting of an absorbed power law. The photon index was left free in a narrow band. Without adding systematic errors in the spectra, the reduced chisquared (hereafter, $\chi_{\text {red }}^{2}$ ) was well above 1.0. When including a $0.8 \%$ systematic error, the $\chi_{\text {red }}^{2}$ dropped to 1.0: this level was adopted for XTE J1818-245 to account for uncertainties in the PCA response. This value is consistent with Jahoda et al. (2006).

Data from the High-Energy Timing Experiment (HEXTE) were reduced in the standard way as described in Rodriguez et al. (2003) apart from the newer version of the reduction software. Due to problems brought about by the rocking motion of HEXTE Cluster A, the extraction of spectra was restricted to Cluster B. Furthermore, due to poor statistics in the HEXTE data points in most of the observations, all channels were rebinned by a factor of 4 . The resultant RXTE spectra of a single observation were fitted simultaneously between $3-25 \mathrm{keV}$ for PCA and 20-150 keV for HEXTE. Note that we also added the IBIS/ISGRI data when available; one INTEGRAL revolution corresponds typically to three distinct $R X T E$ observations.

\subsection{Swift/XRT}

After the observation that announced a refined X-ray Swift position (Still et al. 2005), an additional Swift observation took place on 2005 August 20. The source was observed for 129 s in photon counting mode in order to assess its position. The XRT data were processed with the xrtpipeline (v0.9.9) task applying standard calibration, filtering and screening criteria. An on board event threshold of $\sim 0.2 \mathrm{keV}$ was applied to the central pixel of 


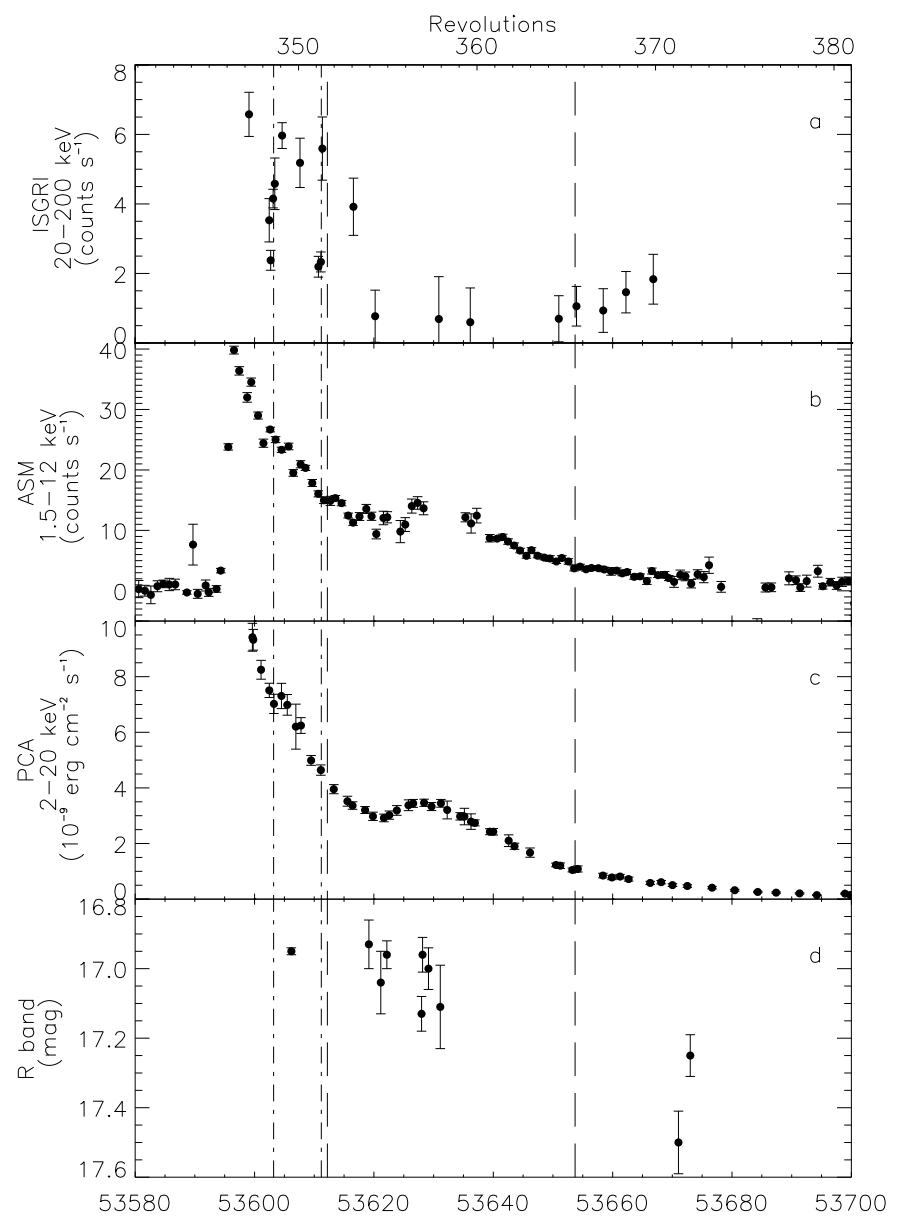

Fig. 3. Light curves of XTE J1818-245 at several wavelengths. a) ISGRI light curve (INTEGRAL revolutions indicated above); b), c) $R X T E / A S M$ and PCA light curves (flux obtained from spectral modeling, see Sect. 3.4); d) REM and NTT light curves in the $R$ filter (magnitudes de-reddened). Dot-dashed lines correspond to the radio peaks at $4.9 \mathrm{GHz}$, then at $1.4 \mathrm{GHz}$ (during our coverage) while dashed lines locate the spectral transitions from SIMS to HSS and then back to the SIMS.

each event, which has been proven to reduce most of the background due to either the bright Earth limb or the CCD dark current (which depends on the CCD temperature). For our analysis, XRT grades in the $0-12$ range were selected. Since the source was extremely bright with more than 50 counts $\mathrm{s}^{-1}$, the data suffered from heavy pile-up. In order to overcome this problem, we extracted photons from an annular region with inner and outer radius of 25 and 80 pixels respectively. 3831 photons were extracted in the $0.3-10 \mathrm{keV}$ energy band and used in the spectral analysis.

\subsection{Optical and NIR}

Optical and NIR observations were performed with the Rapid Eye Mount (REM) telescope (Zerbi et al. 2001; Chincarini et al. 2003; Covino et al. 2004) equipped with the ROSS optical spectrograph/imager and the REMIR NIR camera. Observations of XTE J1818-245 were carried out during 2005 SeptemberOctober ( $R$ band), and on October 29 (JHK bands); late time observations in the $R$ band were also performed during quiescence on 2007 July 18 . Image reduction was carried out by following the standard procedures: subtraction of an averaged bias frame and division by a normalized flat frame. Astrometry was performed using the USNOB $1.0^{1}$ and the $2 \mathrm{MASS}^{2}$ catalogues. Aperture photometry performed with the SExtractor package (Bertin \& Arnouts 1996) for all the objects in the field. The calibration was done against Landolt standard stars for the optical filters and against the 2MASS catalog for the NIR filters. In order to minimize any systematic effect, we performed differential photometry with respect to a selection of local isolated and non-saturated standard stars. Due to the low galactic latitude $\left(-4^{\circ}\right)$ and relatively low absorption in the line of sight, doing this analysis presented a number of challenges in obtaining the absolute flux calibration (difficulties in finding a sourcefree background annulus). The counterpart to XTE J1818-245 was sufficiently isolated from neighboring stars and we verified that the corrected light curves of the comparison field stars were sufficiently stable (within the errors of $\sim 0.05 \mathrm{mag}$ ).

The source was also observed in the optical and NIR with the imager SUSI-2 installed on the NTT (New Technology Telescope) at La Silla Observatory (private communication: S. Chaty). In this paper, we include only the $U, B, V, R$ and $I$ photometry to build the Spectral Energy Distributions (hereafter $\mathrm{SED})$ simultaneously with the radio/X-ray $/ \gamma$-ray data.

\subsection{Radio}

\subsubsection{VLA}

We observed XTE J1818-245 with the National Radio Astronomy Observatory (NRAO) VLA at 1.4, 4.9, 8.4 and $15 \mathrm{GHz}$ from 23:34 UT of August 22 to 01:35 UT of August 23 with the VLA in its C configuration. These observations were thus simultaneous with our INTEGRAL run. The receiver setup included two intermediate frequency (IF) channel pairs of $50 \mathrm{MHz}$ bandwidth each. We used the phase reference calibrators $\mathrm{J} 1811-2055$ at $1.4 \mathrm{GHz}, \mathrm{J} 1820-2528$ at 4.9 and $8.4 \mathrm{GHz}$, and J1751-2524 at $15 \mathrm{GHz}$. The flux density calibrator was $\mathrm{J} 1331+3030$ (3C 286). Snapshots of $10 \mathrm{~min}$ were obtained at $1.4,4.9$ and $15 \mathrm{GHz}$. We also acquired $\mathrm{a} \sim 1 \mathrm{~h}$ light curve at $8.4 \mathrm{GHz}$.

Archival data obtained with the VLA in the same configuration were also retrieved from the NRAO database. A log of the observations, the observed fluxes and their associated errors can be found in Table 2. All the data were reduced using standard procedures within the NRAO aips software package. We restricted the $1.4 \mathrm{GHz}$ data analysis to baselines above $3 \mathrm{k} \lambda$ to avoid the extended galactic diffuse emission as much as possible. Images with natural weighting were produced and flux density measurements were obtained with the aips task JMFIT: the absolute flux calibration is expected to be accurate to the $3 \%$ level.

\subsubsection{VLBA}

From 01:00 to 04:00 UT of August 23, we also observed XTE J1818-245 with the NRAO Very Long Baseline Array (VLBA) simultaneously at 2.3 and $8.4 \mathrm{GHz}$. These observations were simultaneous with our INTEGRAL run and overlapped with the end of the VLA observations. They were conducted using the phase-referencing technique, switching between the phase reference calibrator J1820-2528 and XTE J1818-245(separated by $1^{\circ}$ ), with cycling times of $2.5 \mathrm{~min}$ (100 s for the source and $50 \mathrm{~s}$ for the calibrator), compatible with

\footnotetext{
1 http://www . nofs.navy -mil/data/fchpix/

2 http://www . ipac. caltech.edu/2mass/
} 
Table 2. VLA observation log for XTE J1818-245 indicating the calendar dates, MJD and INTEGRAL revolutions during which the source was observed, together with flux densities at four radio frequencies, the radio spectral index $\alpha$, errors and the spectral X-ray state.

\begin{tabular}{|c|c|c|c|c|c|c|c|c|}
\hline $\begin{array}{c}\text { Date } \\
\text { (yyyy-mm-dd) }\end{array}$ & $\begin{array}{l}\text { Time } \\
\text { (MJD) }\end{array}$ & Rev. & $\begin{array}{c}S_{1.4 \mathrm{GHz}} \\
(\mathrm{mJy})\end{array}$ & $\begin{array}{c}S_{4.9 \mathrm{GHz}} \\
(\mathrm{mJy})\end{array}$ & $\begin{array}{c}S_{8.4 \mathrm{GHz}} \\
(\mathrm{mJy})\end{array}$ & $\begin{array}{c}S_{15 \mathrm{GHz}} \\
(\mathrm{mJy})\end{array}$ & $\begin{array}{l}\text { Spectral index } \alpha \\
\left(S_{v} \propto v^{\alpha}\right)\end{array}$ & X-ray state \\
\hline $2005-08-20$ & 53602.10 & 347 & & $7.13 \pm 0.07(\mathrm{i}, 3.5 \sigma)$ & & & & SIMS \\
\hline $2005-08-21$ & 53603.19 & 348 & & $27.33 \pm 0.06(\mathrm{~d}, 7.7 \sigma)$ & & & & SIMS \\
\hline $2005-08-23$ & 53605.02 & 349 & $1.5 \pm 0.4$ & $1.49 \pm 0.07$ & $2.3 \pm 1.0(\mathrm{o}, 25 \sigma)$ & $<0.83$ & $\sim 0$ & SIMS \\
\hline $2005-08-24$ & 53606.19 & 349 & & & $0.60 \pm 0.04$ & & & SIMS \\
\hline $2005-08-27$ & 53609.19 & 350 & & $1.12 \pm 0.07$ & & & & SIMS \\
\hline $2005-08-29$ & 53611.19 & 351 & $9.3 \pm 0.5(\mathrm{~d}, 3.1 \sigma)$ & $3.72 \pm 0.08$ & $2.27 \pm 0.07$ & & $-0.8 \pm 0.1$ & SIMS \\
\hline 2005-09-04 & 53617.09 & 353 & $<1.22$ & $0.77 \pm 0.08$ & $0.40 \pm 0.09$ & & $-1.2 \pm 0.5$ & HSS \\
\hline 2005-09-11 & 53624.11 & & $<0.86$ & $<0.23$ & $<0.17$ & & & HSS \\
\hline 2005-09-18 & 53631.15 & & & $<0.17$ & & & & HSS \\
\hline 2005-09-23 & 53636.12 & & & $<0.13$ & & & & HSS \\
\hline 2005-10-06 & 53649.02 & & & $<0.22$ & & & & HSS \\
\hline 2005-10-13 & 53656.02 & & & $<0.16$ & & & & SIMS \\
\hline 2005-10-17 & 53660.84 & & & $<0.23$ & & & & SIMS \\
\hline $2006-02-26$ & 53792.51 & & & $<0.23$ & & & & Unknown \\
\hline
\end{tabular}

Note:

The large errors at $1.4 \mathrm{GHz}$ are due to bright sources in the primary beam (field of view). Upper limits are at the $3 \sigma$ level. The large errors at $8.4 \mathrm{GHz}$ during these observations (Rev. 349) reflect the variability of the source (see text). For the runs where variability is present, we quote in parentheses whether the source flux density is increasing (i), decreasing (d) or oscillating (o), together with the significance of this variability.

the expected coherence times. Scans of 2 min were acquired on the fringe finders J1733-1304 and J2000-1748. The data were recorded with 2-bit sampling at $256 \mathrm{Mbps}$ at both circular polarizations. A total bandwidth of $64 \mathrm{MHz}$ was provided by 8 subbands. Half of the bandwidth was used at $2.3 \mathrm{GHz}$ and the other half at $8.4 \mathrm{GHz}$. The data were processed at the VLBA correlator in Socorro, using an integration time of 2 s. Post-processing and data reduction were conducted using standard procedures within the NRAO aips software package.

\section{Results}

The following two sub-sections refer to the hard X-ray image shown in Fig. 2 and the optical, (N)IR and $\mathrm{X} / \gamma$-ray light curves shown in Fig. 3.

\subsection{X-ray position}

In the combined IBIS/ISGRI images obtained near the peak of the outburst (August 20-21), XTE J1818-245 was detected at 18 and $12 \sigma$ in the $20-40$ and 40-80 keV energy bands, respectively. Figure 2 shows the 20-40 keV IBIS/ISGRI mosaic obtained during $\sim 70 \mathrm{ks}$ within Rev. 348 . The best-fit position in this image is $\alpha_{\mathrm{J} 2000}=18^{\mathrm{h}} 18^{\mathrm{m}} 24.36$ and $\delta_{\mathrm{J} 2000}=-24^{\circ} 32^{\prime} 34^{\prime \prime} \cdot 3$, with an accuracy of 1.58 at the $90 \%$ confidence level (Gros et al. 2003).

This is compatible within errors with the most precise position obtained from the XRT data using xrtcentroid: $\alpha_{\mathrm{J} 2000}=$ $18^{\mathrm{h}} 18^{\mathrm{m}} 24^{\mathrm{s}} .02$ and $\delta_{\mathrm{J} 2000}=-24^{\circ} 32^{\prime} 19^{\prime} \cdot 3$, with an accuracy of $3^{\prime \prime} .5$ at the $90 \%$ confidence level.

In turn, this lies just $5{ }^{\prime \prime} 8$ away from the center of the radio position at $4.9 \mathrm{GHz}$ reported by Rupen et al. (2005) at the beginning of the outburst: $\alpha_{\mathrm{J} 2000}=18^{\mathrm{h}} 18^{\mathrm{m}} 24^{\mathrm{s}} .43 \pm 0.2$ and $\delta_{\mathrm{J} 2000}=-24^{\circ} 32^{\prime} 17^{\prime \prime} .96 \pm 00^{\prime} 4$ (see Sect. 3.5.2 for an updated radio position). The high-energy source and the optical/radio counterparts are therefore all unambiguously associated with the new $\mathrm{X}$-ray transient source.

\subsection{X-ray and soft $\gamma$-ray light curves}

Figure $3 \mathrm{a}$ shows the INTEGRAL/IBIS light curve of XTE J1818-245 during the outburst. The peak seen by ISGRI occurs at approximately MJD 53599: the source flux reaches the maximum value of $\sim 7$ counts $\mathrm{s}^{-1}$ in the $20-200 \mathrm{keV}$ range, which corresponds to $\sim 33 \mathrm{mCrab}$. Note that due to incomplete ISGRI coverage at the beginning of the outburst, the actual hard $\mathrm{X}$-ray maximum may have occurred a few days earlier. Up to MJD 53620, the IBIS/ISGRI count rate slowly decreased.

Figure $3 \mathrm{~b}$ shows the $1.5-12 \mathrm{keV}$ RXTE/ASM daily average light curve from (2005) July 29 to December 16, with a peak flux around MJD 53596.5. Assuming an exponential shape for both the rise and the decay phases seen in the ASM light curve, we obtained time constants of, respectively, $5 \pm 1$ and $19 \pm 1$ days. An interesting feature is present between MJD 53620 and 53635 (Fig. 3c): the exponential decrease of the 2-20 keV flux ceases, and then begins to increase again during the subsequent $\sim 10$ days. A shorter event, with smaller amplitude, might have also occurred between MJD 53603 and 53606. This feature, sometimes known as a secondary maximum or a bump has already been noticed in several XNe (e.g., A0620-00, Nova Muscae, 4U 1543-47) and will be discussed Sect. 4.1.

\subsection{Hardness intensity diagrams and quasi-periodic oscillations}

To get a first idea of the spectral behaviour of the source, we produced a hardness-intensity diagram (HID) with $R X T E / \mathrm{PCA}$ (Fig. 4) similar to those widely used in the literature (Fender et al. 2004). While there was no coverage from the start of the outburst to the peak, XTE J1818-245 did not follow the usual path of $\mathrm{XNe}$ in outburst on this diagram: although it certainly went from high flux and relatively low HR to lower flux and higher HR, tracing the end of the usual Q-shape (see, e.g., Belloni et al. 2005), there were slight deviations. Indeed, the source returned along its path twice and traced a $\mathrm{Z}$ pattern in each spectral state, evolving from low to high HR (see Sect. 4.1).

Using this HID obtained with the PCA, together with the analysis that will follow (Sect. 3.4, Figs. 5 and 6), we identified 


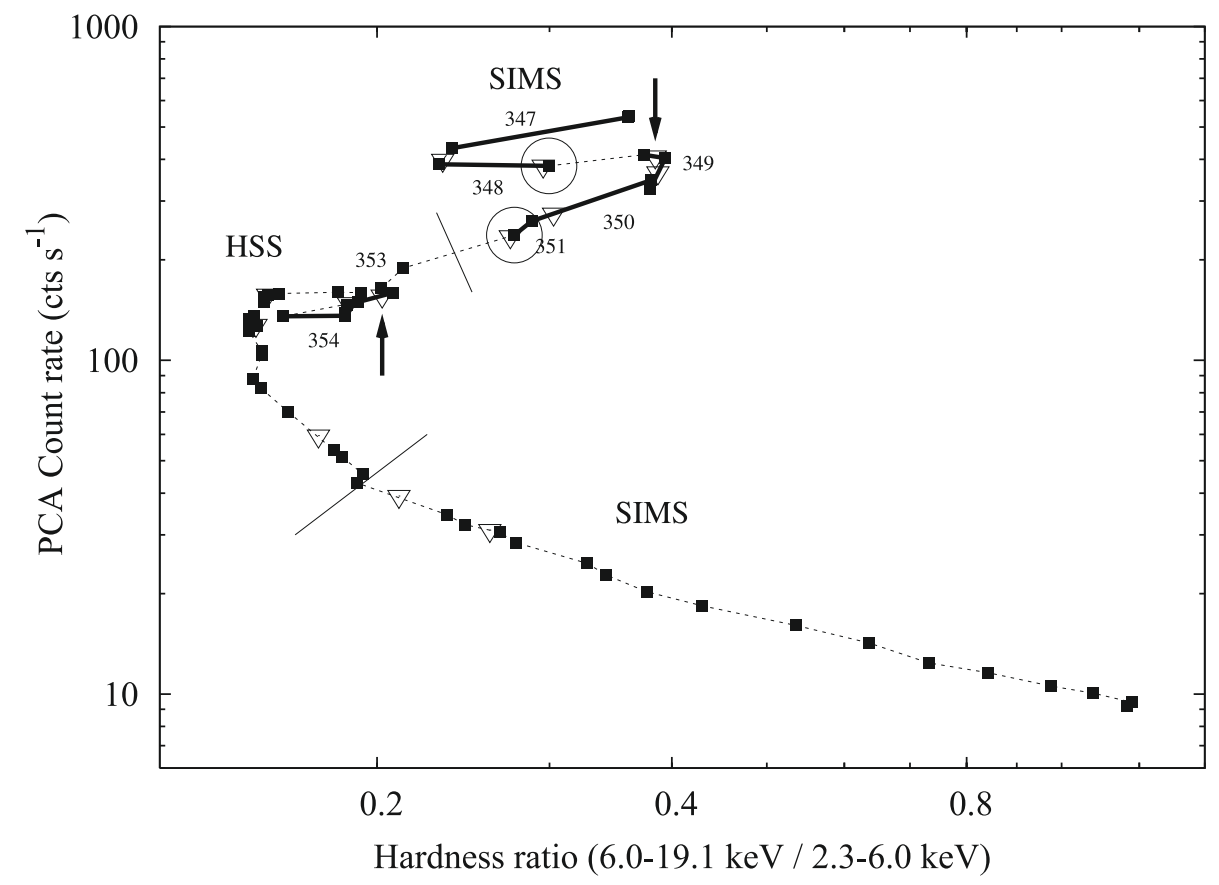

Fig. 4. Hardness-intensity diagram of XTE J1818-245 on a logarithmic scale as obtained with the top layer of RXTE/PCA (2.3-19.1 keV, detector 2). The source globally evolved from top to bottom and from right to left then right again, but returned along the track twice. The filled squares represent individual RXTE observations, the thicker paths indicate the times of the INTEGRAL Rev. (approximately indicated). The SIMS and HSS states are delimited by solid lines perpendicular to the source path. The times of radio observations are represented by the open triangles. The big circles correspond to the times of the radio flares (top: $4.9 \mathrm{GHz}$; bottom: $1.4 \mathrm{GHz}$ ). The downward arrow on top indicates the HID position during our simultaneous radio and ToO observations of MJD 53605 (Rev. 349). The upward arrow marks the HID position during the HSS radio detection on MJD 53617.

two distinct spectral states. The upper branch on the HID had a HR $\sim 0.3$, a power law flux representing $\sim 10-20 \%$ of the total 2-20 keV flux, and a spectral index in the range 2.2-2.5 (Fig. 7). This Soft Intermediate State (SIMS) evolved to a pure HSS around MJD 53612: the power law fraction dropped to $\sim 5 \%$, with a HR around 0.15. After MJD 53654, the source returned to the SIMS, and slowly evolved to harder states: the power law fraction increased again to $20 \%$, while the HR increased to 1 , and the spectral index gradually decreased. Unfortunately, the X-ray coverage did not extend beyond MJD 53700, and thus there is no proof that the source eventually evolved to the LHS (the photon index was $\sim 2.3$ instead of 1.3-1.5 and the power law fraction was too low compared to the high values - greater than $80 \%$ - reported in Homan \& Belloni 2005; see Table 3), as is usually the case for such objects (e.g., Cadolle Bel et al. 2004; McClintock \& Remillard 2006). In this work, the last and hardest data we have analysed occur when the source was still in the SIMS.

The RXTE PDS showed very weak variability, with the power decreasing against frequency in a roughly power law-like fashion below a few $\mathrm{Hz}$. The total RMS level was around 6-8\% before MJD 53608 and too faint (below the detector noise) to be quantified. Above a few $\mathrm{Hz}$, the PDS quickly fell to the level of the white noise. QPOs were also searched for, but none were detected. To quantify this, we used the following formula from van der Klis (2006) which reflects the detection limit at which a QPO would be picked up at the $3 \sigma$ level:

$A=\left(6 * \frac{S+B}{S^{2}} \sqrt{\frac{F W H M}{T}}\right)^{\frac{1}{2}}$ where $A$ is the detection level (at $3 \sigma$ ), $S$ the source count rate, $B$ the background level, FWHM the width of the searched QPO and $T$ the exposure time in seconds. In the case of XTE J1818-245, for a typical QPO with a $1 \mathrm{~Hz} F W H M$, the limit at which a QPO would have been detected at the $3 \sigma$ level is $2 \%$ rms amplitude at the onset of the outburst, and $8 \%$ when the X-ray flux was minimum (at the end of the outburst). The absence of strong QPOs and of strong band-limited noise is expected during soft states like the HSS and the SIMS, giving further support to the proposed spectral classification below.

\subsection{X-ray and $\gamma$-ray spectra}

Using XSPEC v11.3.2 (Arnaud 1996), we fitted spectra from the PCA (3-25 keV), HEXTE (20-100 keV), IBIS (18-200 keV) and XRT (0.3-10 keV) instruments simultaneously. Several models were tested when analyzing the spectra. A normalization constant was added to account for uncertainties in the crosscalibration of the instruments.

The data were well fitted using an absorbed power law combined with a multicolour black-body and a fluorescent (Gaussian) iron line. Neither a reflection component nor a highenergy cut-off were needed at any time in our fits, at least up to $150 \mathrm{keV}$ : this was tested by adding these additional contributions, and, as we did not observe any improvement in the goodness of the fit, we concluded they were not necessary. The absorption was fixed to the value found by XRT $\left(N_{\mathrm{H}}=\right.$ $5.4_{-0.9}^{+2.0} \times 10^{21} \mathrm{~cm}^{-2}$ ) since it cannot be constrained by either the PCA or JEM-X data. This value is compatible, within the errors, with the average galactic column density in the source direction $\left(4.8 \times 10^{21} \mathrm{~cm}^{-2}\right.$, estimated from Schlegel et al. 1998). An iron 


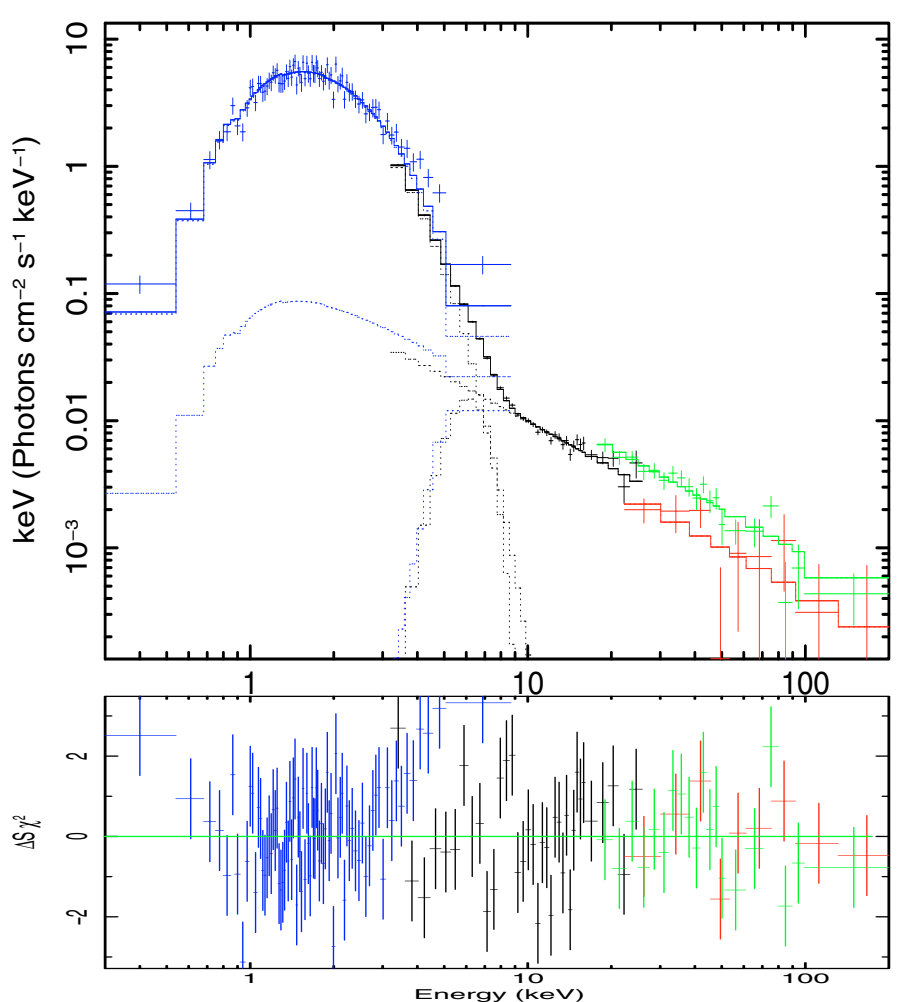

Fig. 5. Energy spectra of XTE J1818-245 during the first INTEGRAL ToO (Rev. 348, MJD 53602.5) with the Swift/XRT (blue), RXTE/PCA (black), RXTE/HEXTE (red) and INTEGRAL/IBIS/ISGRI (green) data, along with the best-fit model: an absorbed multicolour black-body disc and a power law with a Gaussian component (see Table 3 for parameter values). Residuals ( $\Delta S$ $\left.\chi^{2}\right)$ in $\sigma$ units are also shown below. The source was in the SIMS.

line was necessary to adequately fit the data, but the line centroids could not be constrained by the PCA and we thus forced them to have energies above $6 \mathrm{keV}$.

For the disc component, the EZDISKBB model in XSPEC notation (Zimmerman et al. 2005) was used: it assumes a zero-torque boundary condition at the inner edge of the accretion disc, which is not the case in the widely used DISKBB model. This condition normally leads to a more accurate determination of the inner radii of the accretion disc, as well as a more physical value for the maximum temperature in the disc. The quality of the fit to the data was similar for the DISKBB and EZDISKBB models: we only found a factor $\sim 2.2$ of difference between the inner radii, which was consistent with the value found by Zimmerman et al. (2005).

The power law component is taken to be a purely phenomenological model and could signal the presence of a compact jet, a corona, or reprocessed hard X-ray emission due to X-ray heating from an extended central source (Hynes 2005). We tried the more physical Comptonization models of Sunyaev \& Titarchuk (1980) and Titarchuk (1994) without success (the fit was worse than with the simpler model). We also applied much more complex models better adapted for such soft states such as COMPPS and EQPAIR, developed respectively by Poutanen \& Svensson (1996) and Coppi (1999) (see Cadolle Bel et al. 2006 for discussions). Unfortunately, the source was not bright enough to constrain the spectral parameters: the resulting $\chi_{\text {red }}^{2}$ did not decrease. Besides, a simple power law fit allowed us to compare more easily the spectral parameters between the bright beginning and the faint end of the outburst.

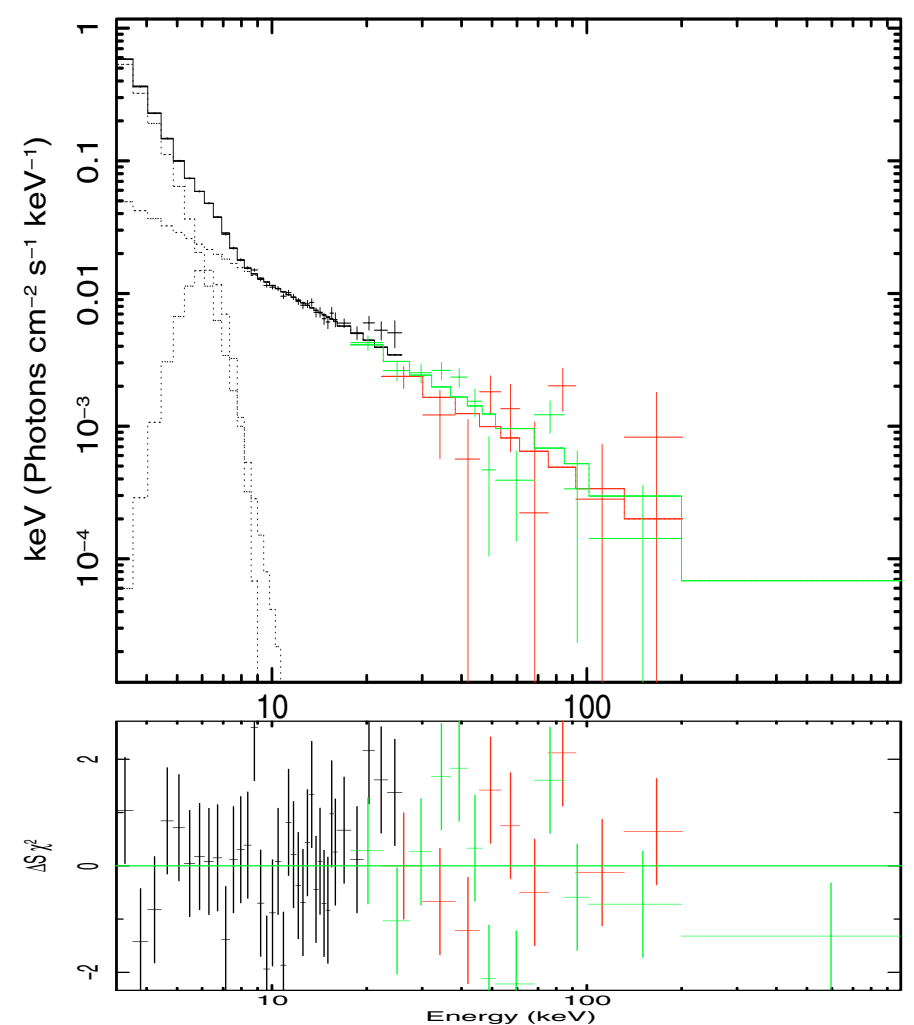

Fig. 6. Energy spectra of XTE J1818-245 during the third INTEGRAL ToO (Rev. 351, MJD 53611.1) with the PCA (black), HEXTE (red) and IBIS/ISGRI (green) data, along with the best-fit model: an absorbed multicolour black-body disc and a power law with a Gaussian component (see Table 3 for parameter values). Residuals $\left(\Delta S \chi^{2}\right)$ in $\sigma$ units are also shown below. The source was in the HSS.

Thus, we fitted all the data with the unique CONSTANT* WABS* (EZDISKBB+GAUSSIAN+POWERLAW) model (in XSPEC terminology) throughout the whole outburst. For all spectra, normalization constants were in the 0.7-1.4 range for instruments other than the PCA (which was fixed to 1). Two examples of fitted spectra obtained during our INTEGRAL ToO observations are shown in Figs. 5 and 6 (in photons $\mathrm{cm}^{-2} \mathrm{~s}^{-1}$ ) with residuals.

Table 3 lists the spectral parameters derived for all the INTEGRAL observations. A plot of the main spectral parameters for all the observations is shown in Fig. 7, together with the ASM light curve. The spectral parameters were compatible with the source being first in the SIMS, then transiting into the HSS and returning to the SIMS, this time with a lower flux and a higher HR; the dashed lines of Fig. 7 indicate both spectral state limits. Throughout the outburst, a regular decrease of the disc temperature from a high value of $\sim 0.64$ to $0.27 \mathrm{keV}$ was observed.

Although not extremely well constrained by the PCA restricted to energies above $3 \mathrm{keV}$, the temperature trend could be derived: we verified that the disc parameters we derived for the XRT data plotted in Fig. 5 are compatible with those epochs for which no XRT data is available. The observed decrease for the inner disc temperature corresponds to what is usually observed for a transient source in outburst: the disc dominates the emission at the beginning and then gradually cools. The rather low temperatures are more consistent with a binary system containing a BH than a neutron star (Tanaka \& Lewin 1995). The disc normalization slowly increased during the outburst, from $\sim 150$ 
Table 3. Best-fit spectral parameters over all our INTEGRAL observations.

\begin{tabular}{|c|c|c|c|c|c|c|c|c|}
\hline $\begin{array}{l}\text { Time } \\
(\mathrm{MJD})\end{array}$ & Observations & Disc Norm. $^{a}$ & $\begin{array}{c}k T_{\text {in }} \\
(\mathrm{keV}) \\
\end{array}$ & $\Gamma$ & $\begin{array}{c}E_{\mathrm{Fe}} \text { line } \\
(\mathrm{keV})\end{array}$ & $\begin{array}{c}\chi_{\text {red }}^{2} \\
\text { (d.o.f.) }\end{array}$ & $\begin{array}{c}F_{\text {total }}^{b} \\
\left(10^{-10} \mathrm{erg} \mathrm{cm}^{-2} \mathrm{~s}^{-1}\right)\end{array}$ & $\begin{array}{c}F_{\text {bolometric }}^{c} \\
\left(10^{-8} \mathrm{erg} \mathrm{cm}^{-2} \mathrm{~s}^{-1}\right)\end{array}$ \\
\hline 53599.8 & Rev. 347 & $1320_{-112}^{+90}$ & $0.640 \pm 0.008$ & $2.44_{-0.07}^{+0.08}$ & $6.0^{+0.05}$ & $1.39(57)$ & $81 \pm 3$ & 5.96 \\
\hline 53602.5 & Rev. 348 & $1990_{-107}^{+21}$ & $0.59 \pm 0.01$ & $2.21_{-0.04}^{+0.04}$ & $6.0^{+0.06}$ & 1.49 (136) & $63 \pm 1$ & 4.19 \\
\hline 53605.5 & Rev. 349 & $1160_{-34}^{+110}$ & $0.624 \pm 0.008$ & $2.34_{-0.08}^{+0.05}$ & $6.0^{+0.04}$ & $1.69(57)$ & $59 \pm 2$ & 3.92 \\
\hline 53607.8 & Rev. 350 & $1090_{-85}^{+100}$ & $0.614 \pm 0.007$ & $2.37_{-0.06}^{+0.07}$ & $6.0^{+0.04}$ & $1.10(48)$ & $52 \pm 2$ & 3.08 \\
\hline 53611.1 & Rev. 351 & $1730_{-130}^{+140}$ & $0.55 \pm 0.007$ & $2.32_{-0.10}^{+0.09}$ & $6.0^{+0.05}$ & $1.51(48)$ & $39 \pm 1$ & 2.20 \\
\hline 53618.5 & Rev. 353 & $2260_{-170}^{+230}$ & $0.51 \pm 0.01$ & $2.30_{-0.21}^{+0.29}$ & $6.0^{+0.07}$ & $0.73(41)$ & $27 \pm 2$ & 2.40 \\
\hline 53619.8 & Rev. 354 & $2270_{-180}^{+230}$ & $0.502 \pm 0.006$ & $2.82_{-0.32}^{+0.58}$ & $6.0^{+0.18}$ & $1.08(41)$ & $25 \pm 1$ & 2.33 \\
\hline 53621.7 & First min. ${ }^{d}$ & $2160_{-140}^{+290}$ & $0.50 \pm 0.01$ & $2.45_{-0.28}^{+0.32}$ & $6.0^{+0.07}$ & $1.41(41)$ & $23.3 \pm 0.5$ & 2.20 \\
\hline 53631.2 & Second. $\max ^{d}$. & $2440_{-206}^{+170}$ & $0.511 \pm 0.005$ & $2.30_{-0.78}^{+0.70}$ & $6.0^{+0.14}$ & $1.08(42)$ & $28.0 \pm 0.5$ & 2.29 \\
\hline 53699.8 & Last obs. ${ }^{d}$ & $<20000$ & $0.27 \pm 0.06$ & $2.28_{-0.24}^{+0.28}$ & - & $1.11(40)$ & $1.5 \pm 0.1$ & 0.92 \\
\hline
\end{tabular}

Notes:

Model applied in XSPEC notations: CONSTANT*WABS*(EZDISKBB+GAUSSIAN+POWERLAW) with $N_{\mathrm{H}}$ fixed to $5.4 \times 10^{21} \mathrm{~cm}^{-2}$. Errors are given at the $90 \%$ confidence level $\left(\Delta \chi^{2}=2.7\right)$.

${ }^{a}$ Disc normalization $K$ is proportional to $(R / D)^{2} \cos \theta$, where $R$ is the inner disc radius in $\mathrm{km}, D$ is the distance to the source in $\mathrm{kpc}$ and $\theta$ the inclination angle of the disc.

${ }^{b}$ Computed in the $2-20 \mathrm{keV}$ range.

${ }^{c}$ Extrapolated in the $0.001 \mathrm{keV}-10 \mathrm{MeV}$ range.

${ }^{d}$ Based on the RXTE/PCA light curve.

to $400 \times D \sqrt{(1 / \cos \theta)} \mathrm{km}$ ( $D$ is the distance to the source in units of $10 \mathrm{kpc}, \theta$ the inclination angle of the disc). Although some caveats may be raised about the exact value of the inner radius of the accretion disc obtained from the fits, a clear trend is seen: the disc receded globally during the decay phase of the outburst. Again, this is a typical feature in X-ray Novae: during the hardening, the disc usually moves outwards slowly (Chen et al. 1997; Cadolle Bel et al. 2004), even though this is not necessarily true for all transient sources (see, e.g., Miller et al. 2006). However, this point is still strongly debated (Done et al. 2007; Rykoff et al. 2007; Gierliński et al. 2008) as, for example, in XTE J1817-330. We do not address this question specifically here as our data - with a lower energy limit of $3 \mathrm{keV}$ - are not sensitive enough for this purpose.

At the same time, at higher energies, the power law photon index varied between 2.0 and 3.5, thereby confirming that the source remained in the softer states. Towards the middle of the observations (MJD 53635-53655) the fraction of the power law flux compared to the total flux decreased from 5 to $2 \%$ (HSS), while the photon indices displayed large error bars (statistical fluctuations). Subsequently, the power law flux increased rapidly from 2 to $5 \%$ and gradually returned to the original $\sim 20 \%$ level (SIMS). The spectral fits and the HR indicate that the spectrum was harder at the end of the outburst compared to the peak. This is not unusual as XNe tend to evolve towards harder states in the late stages of their outbursts. For example, during the decay of their outbursts, XTE J1720-318, XTE J1650-500, SWIFT J1753.5-0127 and GX 339-4 showed a slowly receding disc with decreasing inner temperature, while at the same time the relative amount of the power law contribution increased.

\subsection{Multiwavelength studies}

\subsubsection{NIR/optical}

The standard photometry performed with EMMI/NTT on XTE J1818-245 around August 24 (between 3-5 UT, i.e., Rev. 349) provided the following optical magnitudes (private communication: S. Chaty): $U=18.24 \pm 0.03, B=18.47 \pm 0.014$,
$V=17.46 \pm 0.01, R=16.95 \pm 0.01$ and $I=16.34 \pm 0.01$. They were perfectly consistent with the data reported by Steeghs et al. (2005) and the REM results which cover a much longer period.

Figure $3 \mathrm{~d}$ shows the magnitude in the $R$ filter corrected for reddening, obtained with the REM and NTT telescopes along the outburst. To estimate the $E_{B-V}$ parameter necessary for the de-reddening, we used the relation $N_{\mathrm{H}} / E_{B-V}=$ $5.8 \times 10^{21} \mathrm{~cm}^{-2} \mathrm{mag}^{-1}$ (Bohlin et al. 1978) and the absorption column density estimated from $S_{\text {wift/XRT data }}$ $\left(N_{\mathrm{H}}=5.4_{-0.9}^{+2.0} \times 10^{21} \mathrm{~cm}^{-2}\right)$. The resulting colour excess was $E_{B-V}=0.93_{-0.15}^{+0.34} \mathrm{mag}$. Using a standard extinction curve from Fitzpatrick (1999), we thus obtained the de-reddening parameters for each of the optical/NIR filters.

The source faded consistently, as expected given that the REM observations started during the decay phase of the outburst. Some two months after the peak of the outburst (around MJD 53672), the magnitudes in the $R, J, H$ and $K$ filters were respectively: $>18.3$ (at the $3 \sigma$ limit), $16.2 \pm 0.3,14.7 \pm 0.2$ and $15.1 \pm 0.2$. This is compatible with the classical picture of $\mathrm{XN}$ transients in outburst: well after the peak of the outburst, when the soft thermal component has faded, the accretion disc still contributes (albeit marginally) to the emission in the optical/NIR while the jet activity may have ceased. In quiescence, the optical/NIR is mainly the quiescent, likely optically thin disc, plus the mass donor star, and eventually synchrotron emission from a persistent jet: see, e.g., SWIFT J1753.5-0127 (Cadolle Bel et al. 2007, although this source remained in the LHS), and more generally BH XNe in outburst (Chen et al. 1997; Brocksopp et al. 2001; Hynes et al. 2002) as discussed hereafter.

\subsubsection{Radio}

We show in Table 2 the flux densities measured during the VLA observations from MJD 53602.10-53792.51. The large errors at $1.4 \mathrm{GHz}$ are due to the presence of bright radio sources in the field. The radio light curves at 1.4, 4.9 and $8.4 \mathrm{GHz}$ are shown together with the ASM X-ray light curve in Fig. 8.

At least two distinct radio flares were visible (indicated by dot-dashed lines in Figs. 3, 7 and 8) during the period covered by 


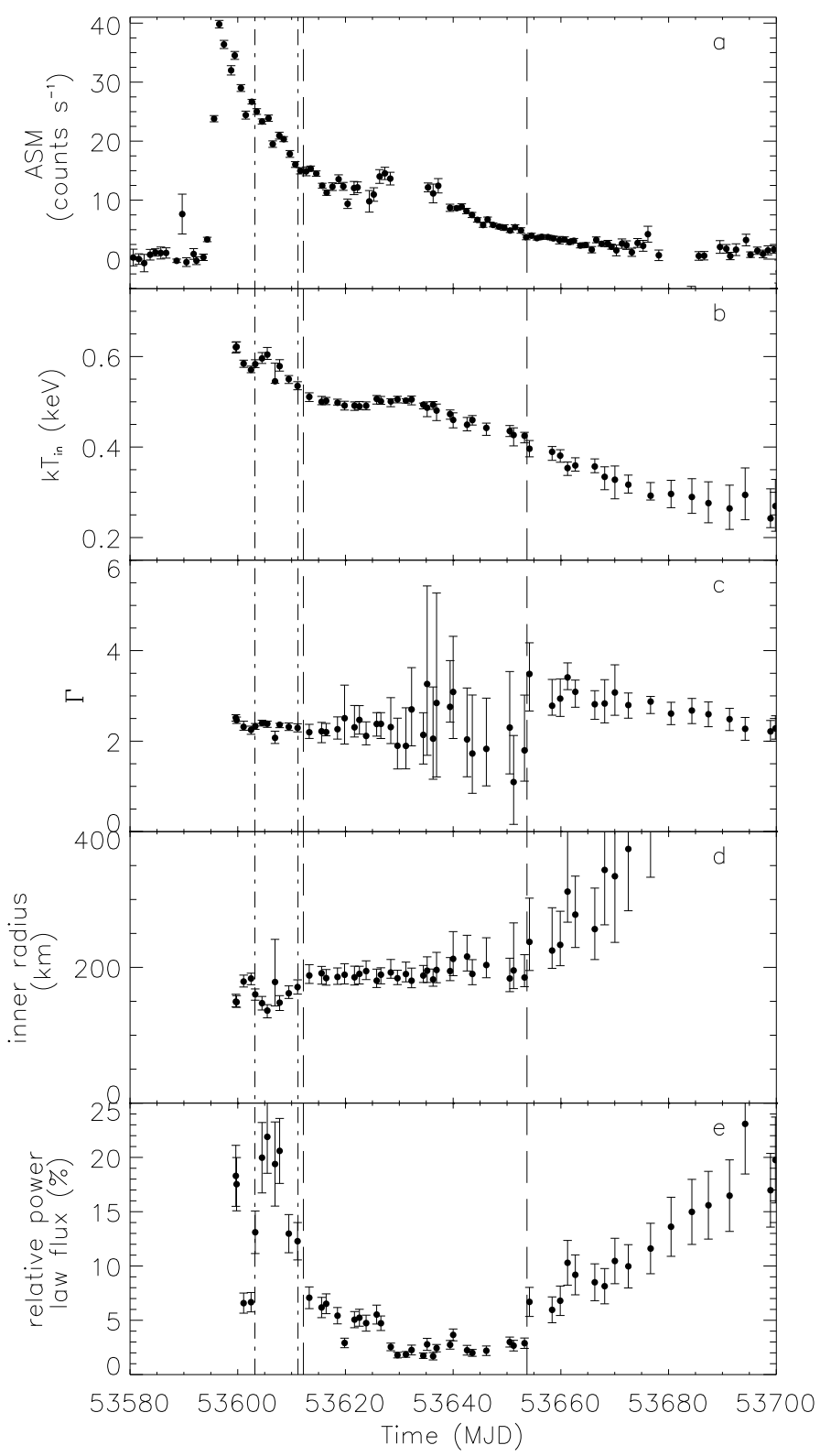

Fig. 7. Spectral characteristics of XTE J1818-245 during outburst; a: the ASM light curve is reproduced here in order to easily compare with the changes in the spectral parameters; b), c), d) disc temperature (in $\mathrm{keV}$ ), photon index and inner radius of the accretion disc (in $\mathrm{km}$ ) derived from the spectral analysis (the inner radius values after MJD 53680 were ignored because of the high level of uncertainties); e: fraction of the power law flux to the total flux in the $2-20 \mathrm{keV}$ range. Dot-dashed lines correspond to the radio flares at 4.9 and $1.4 \mathrm{GHz}$ while dashed lines indicate the spectral transitions from and to the SIMS (see Table 3 and text for details).

our data. First, between MJD 53602 and 53603, XTE J1818-245 showed a strong variation at $4.9 \mathrm{GHz}$ : the flux density increased from $\sim 7$ to $27 \mathrm{mJy}$ in less than $24 \mathrm{~h}$ (Rupen et al. 2005). Afterwards, between MJD 53605 and 53611, the flux density at $1.4 \mathrm{GHz}$ increased from 1.5 to $9.3 \mathrm{mJy}$, while at $8.4 \mathrm{GHz}$ it only increased from 1.5 to $3.7 \mathrm{mJy}$. Meanwhile, small spectral changes were occurring in the X-ray range: XTE J1818-245 was slowly going from a SIMS to a pure HSS without following the classical path in the HID. The X-ray photon index $\Gamma$ was $\sim 2.3$ at that time.

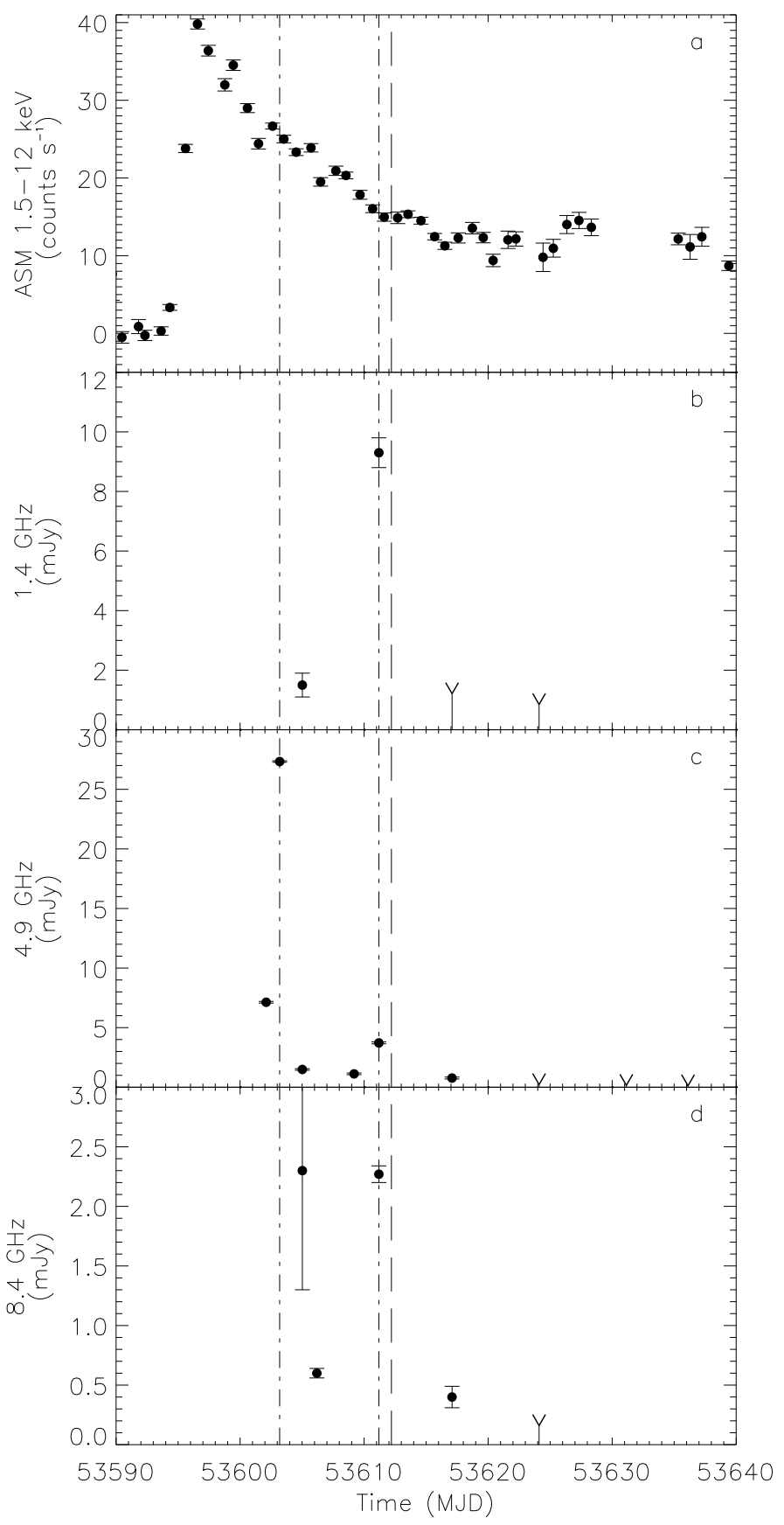

Fig. 8. From top to bottom: zoom on the ASM X-ray light curve (a) and radio flux densities at three frequencies $(1.4,4.9$ and $8.4 \mathrm{GHz}$, respectively in panels b, c and d) obtained with the VLA from MJD 53590 to 53640 . Dot-dashed lines correspond to the radio flares at 4.9 and $1.4 \mathrm{GHz}$, while the dashed lines indicate the spectral transition from the SIMS to the HSS and then back to SIMS.

Regarding the observations conducted on 2005 August 23 (MJD 53605.02) simultaneously with the INTEGRAL ones (Rev. 349), the $8.4 \mathrm{GHz}$ flux density measured in the image obtained with the whole data set was $2.37 \pm 0.04 \mathrm{mJy}$. We split the data set in 10 and 5 minute intervals, constructed images and measured the corresponding flux densities to search for variability. The results are shown in Fig. 9. As can be seen, the flux density at $8.4 \mathrm{GHz}$ increased from 1 to $4 \mathrm{mJy}$ in just 30 min and decreased again to the initial value on a similar timescale. Meanwhile, the phase-reference source J1820-2528, observed every $10 \mathrm{~min}$, was clearly stable (with a less than 


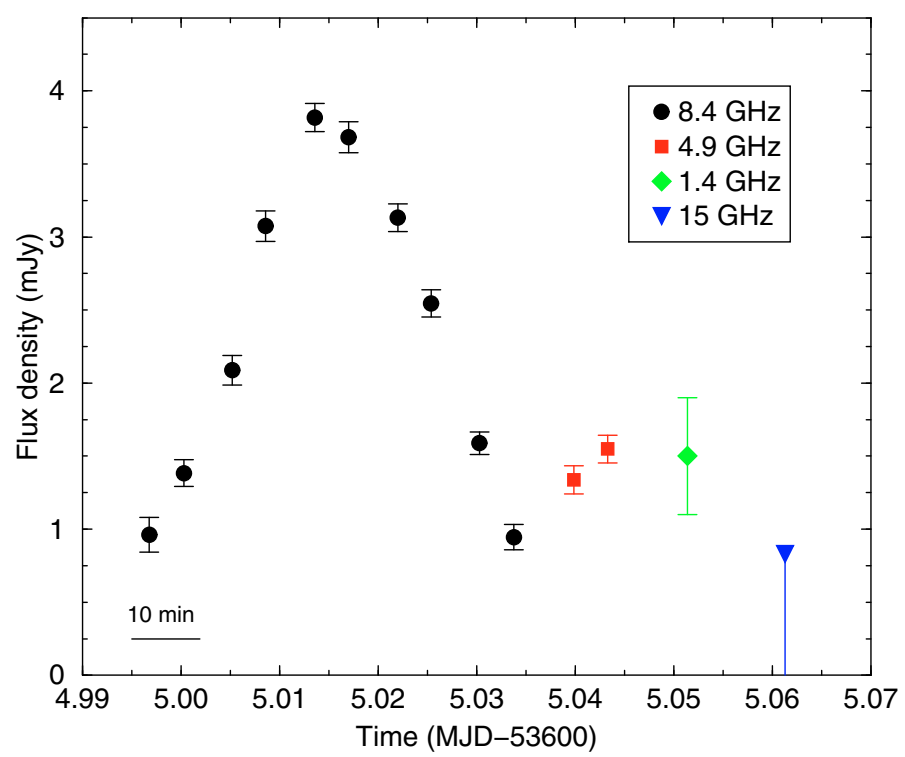

Fig. 9. Radio flux density measurements as a function of time obtained on 2005 August 23 at 8.4 (circles), 4.9 (squares), 1.4 (diamond) and $15 \mathrm{GHz}$ (lower triangle). The bin size is 5 minutes for the first two frequencies and 10 minutes for the other ones. The $15 \mathrm{GHz}$ data point corresponds to the $3 \sigma$ upper limit.

$7 \%$ probability that the source was variable): this showed that XTE J1818-245 was variable. The average of the 5-min measurements is $2.3 \pm 1.0 \mathrm{mJy}$, and this is the value we report in Table 2. Since the source was highly variable and the multi-frequency radio data were not strictly simultaneous, it was not possible to derive a reliable spectral index for the source. We analysed separately the flux densities measured in different IFs for the 10 min scans around the maximum at $8.4 \mathrm{GHz}$, but the large errors did not allow us to extract any conclusion on the spectral shape. However, comparing the quasi-simultaneous 8.4 and $4.9 \mathrm{GHz}$ data in Fig. 9, one can see that the spectrum was most likely close to flat.

A similar variability analysis splitting the data of each run was conducted for the runs with flux densities above $1 \mathrm{mJy}$. Variability can only be claimed in the following four cases (see also the caption of Table 2). The source flux density at $4.9 \mathrm{GHz}$ was increasing during the first radio observation, with a significance of $3.5 \sigma$. It was already decreasing $(7.7 \sigma)$ during the second one, when the first radio outburst took place. It was oscillating $(25 \sigma)$ at $8.4 \mathrm{GHz}$ during the third run, as noted in the above paragraph. It was marginally decreasing $(3.1 \sigma)$ at $1.4 \mathrm{GHz}$ at the time of the second radio outburst.

The radio position reported by Rupen et al. (2005) was obtained at $4.9 \mathrm{GHz}$ at the beginning of the outburst: however, at that time, the source was highly variable and the flux density decreased from $28.1 \pm 0.1$ to $26.7 \pm 0.2 \mathrm{mJy}$ in just $20 \mathrm{~min}$ of observations. Therefore, to obtain the best position of the radio source, we selected the VLA $8.4 \mathrm{GHz}$ data set acquired when the source was brighter and stable, which corresponds to 2005 August 29 (MJD 53611.19). The best-fit position was $\alpha_{\mathrm{J} 2000}=$ $18^{\mathrm{h}} 18^{\mathrm{m}} 24^{\mathrm{s}} .430 \pm 0.003$ and $\delta_{\mathrm{J} 2000}=-24^{\circ} 32^{\prime} 17^{\prime \prime} .91 \pm 00^{\prime} 07$. This position is more precise than (and compatible within the errors with) the one reported by Rupen et al. (2005). The offset is 0 .' 42 only from the R-band optical position reported by Steeghs et al. (2005) and 0.'49 from our REM $R$-band results, both given with an estimated uncertainty around, respectively, 0.3 and 0.2 . Therefore, these optical positions are fully compatible, within the errors, with our radio results. The radio source was unresolved at all frequencies and epochs.

The VLBA observations conducted at the end of our simultaneous VLA run show that no source was detected within $1^{\prime \prime}$ of the VLA position of XTE J1818-245. We could set an upper limit of $1.0 \mathrm{mJy} \mathrm{beam}^{-1}$ at both 2.3 and $8.4 \mathrm{GHz}$ frequencies. The beam at $2.3 \mathrm{GHz}$ was at $11.6 \times 5.9^{\prime \prime}$ with a position angle (north to east) of $-5^{\circ}$ while the beam at $8.4 \mathrm{GHz}$ was at $2.5 \times 1.5^{\prime \prime}$ with a position angle of $-3^{\circ}$.

\section{Discussion}

\subsection{X-rays and $\gamma$-rays}

In Sects. 3.3 and 3.4, we showed that, during the broad-band observations of XTE J1818-245 presented in this work, the source switched between the SIMS and the HSS, as shown by the bestfit parameters obtained in Table 3 and summarized in Fig. 7. In the X-ray range, the high peak luminosity, the fast rise timescale, the bright state and the spectral properties are typically observed in other dynamically confirmed BHs during their outbursts. The characteristic decay time derived is compatible with the usual behaviour of XNe in outburst (Tanaka \& Shibazaki 1996; Chen et al. 1997) as seen recently in XTE J1720-318 (Cadolle Bel et al. 2004) and SWIFT J1753.5-0127 (Cadolle Bel et al. 2007). This clearly supports the hypothesis that XTE J1818-245 is a good BHC.

Interestingly, secondary maxima or bumps were seen in the ASM and PCA light curves: a smaller one around MJD 53605 (just before the first observed radio outburst when the disc temperature was at a relative minimum) and a clear one around MJD 53625 (Fig. 7). During those bumps, the X-ray flux levelled off and was accompanied by a simultaneous increase in the disc temperature (Fig. 7). Several mechanisms have been proposed to explain similar features, one of the most promising being the outer disc irradiation proposed by King \& Ritter (1998). In this model, the central X-ray source irradiates the accretion disc, and maintains it hot until the central accretion rate is sharply reduced. Thus, part of the disc keeps a high effective viscosity during the outburst. If the irradiation is strong enough to heat the whole disc, the X-ray light curve will decay exponentially.

Moreover, in this picture, the secondary maxima are explained by a more marked increase in viscosity in the outer regions than further in, causing a pulse of extra mass to move inwards. Depending on the radial size of the disc region that is involved, the secondary maximum thus appears roughly on a viscous time-scale after the initial outburst.

Using the model developed in Shahbaz et al. (1998), one can try to estimate the distance to the source with the following formula:

$D_{\mathrm{kpc}}=4.3 \times 10^{-5} \sqrt{\frac{\eta f t_{\mathrm{s}}^{3}}{F_{\mathrm{p}} \tau_{\mathrm{d}}}}$

$\eta$ is the radiation efficiency parameter (typically 0.10 for BH systems); $f$ is the ratio of the disc mass at the start of the outburst to the maximum possible (we took 0.5 and 1 ); $t_{\mathrm{s}}$ is the time in days when the secondary maximum occurs after the outburst peak; $F_{\mathrm{p}}$ is the peak flux in the $0.4-10 \mathrm{keV}$ band, and $\tau_{\mathrm{d}}$ is the e-folding time of the decay in days. For XTE J1818-245, we obtained $\tau_{\mathrm{d}}=18-20 \mathrm{~d}, t_{\mathrm{s}} \approx 30 \mathrm{~d}$ and a distance between $2.8-4.3 \mathrm{kpc}$. We used it in the luminosity estimation, since this is one of the few constraints we have on the source distance which relies on 


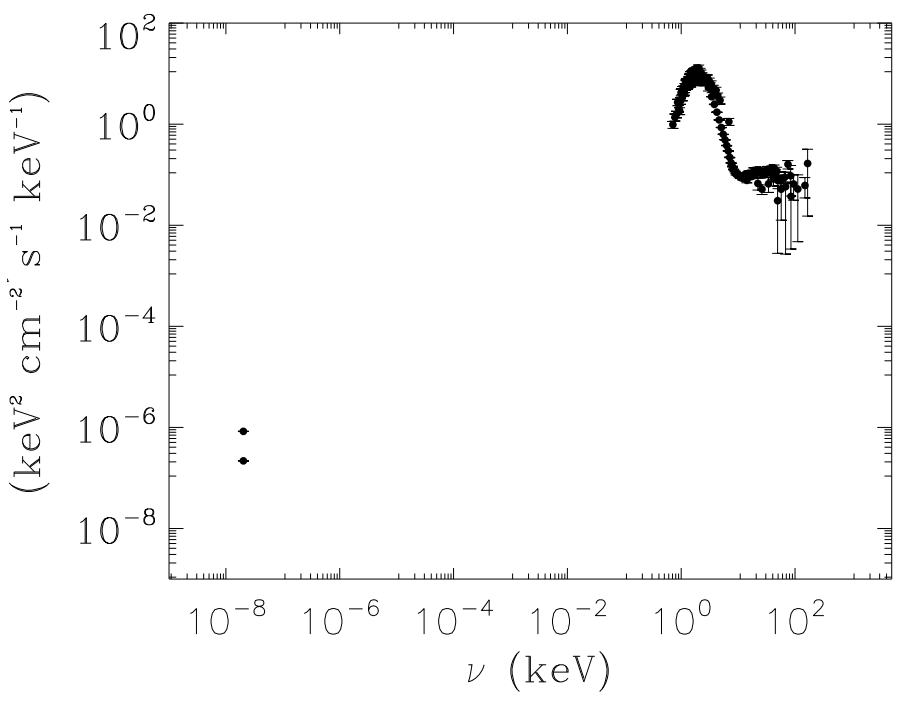

Fig. 10. Unabsorbed spectral energy distribution of XTE J1818-245 from radio (VLA at $4.9 \mathrm{GHz}$ ) to X-ray/soft $\gamma$-ray data (XRT, PCA, HEXTE and ISGRI from 0.3 up to $200 \mathrm{keV}$ ) during the end of Rev. 347/beginning of 348 (i.e., first INTEGRAL ToO). A strong increase in the VLA flux density was observed in less than 1 day.

a model. This model also leads to the determination of the outer radius of the disc $\left(2.5-4.1 \times 10^{10} \mathrm{~cm}\right)$ and its outer viscosity $\left(0.8-2.3 \times 10^{14} \mathrm{~cm}^{2} \mathrm{~s}^{-1}\right)$. It should be noted, however, that this model has been tested on $\sim 10$ sources only, and should not be considered as a precise measurement of these parameters.

The evolution of the source in the HID (Fig. 4) did not strictly follow the usual Q-path of $\mathrm{BH} \mathrm{XNe}$ : between its first time in the SIMS and HSS, the source returned twice along the usual path towards higher HR, tracing a small Z-shape, before going back to a smoother evolution in the last part of the SIMS. This behaviour was first seen in XTE J1550-564 by Homan et al. (2001), and that led these authors to suggest the need for an additional parameter to the accretion rate to drive state changes.

While our spectral information usually started above $3 \mathrm{keV}$, leading to a possible underestimation of the bolometric luminosity, we derived for the spectra obtained during the first INTEGRAL ToO with Swift/XRT a high unabsorbed $0.3-10 \mathrm{keV}$ flux of $3.8 \times 10^{-8} \mathrm{erg} \mathrm{cm}^{-2} \mathrm{~s}^{-1}$. The $10-200 \mathrm{keV}$ flux reached $2.6 \times 10^{-9} \mathrm{erg} \mathrm{cm}^{-2} \mathrm{~s}^{-1}$ while the bolometric flux (extrapolated from $0.001 \mathrm{keV}$ to $10 \mathrm{MeV}$ ) was $4.2 \times$ $10^{-8} \mathrm{erg} \mathrm{cm}^{-2} \mathrm{~s}^{-1}$ (dominated by the disc emission at the beginning of the outburst). Assuming a distance to the source of between $2.8-4.3 \mathrm{kpc}$, this corresponds to an unabsorbed bolometric luminosity of $0.4-0.9 \times 10^{38} \mathrm{erg} \mathrm{s}^{-1}$, below the Eddington regime for a 3 solar mass $\mathrm{BH}$ (i.e., $3.9 \times 10^{38} \mathrm{erg} \mathrm{s}^{-1}$ ). This high luminosity is not surprising considering the soft spectral state at this time. The disc and bolometric fluxes then decreased while the high-energy emission, well modeled by a power law, increased up to $25 \%$ (see Table 3 and Fig. 7).

In the latest stages of the observations presented in this work, the source flux was still higher than the expected flux in the LHS: the source stayed far from the "hard section" zone of the HID. The relatively high power law index of $\sim 2.3$, its low flux contribution and the HR value in the HID clearly show the source had not reached the LHS yet; extrapolating the later behaviour in Fig. 4 shows that it could have taken weeks before the source did so. Until the end of our observations, the luminosity stayed greater than the usual quiescence values (between

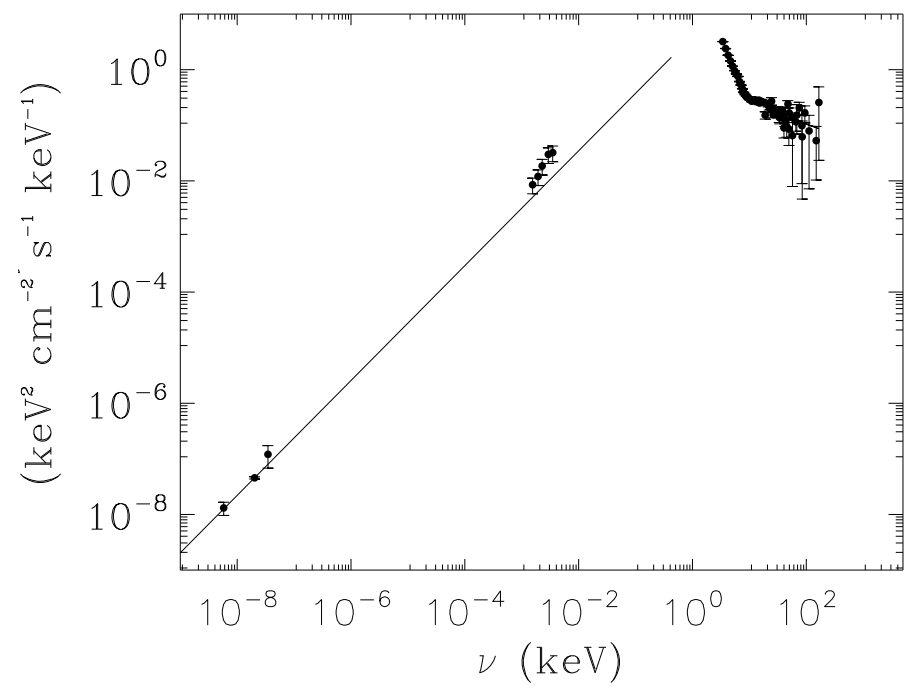

Fig. 11. Unabsorbed spectral energy distribution of XTE J1818-245 including radio (VLA at 1.4, 4.9 and $8.4 \mathrm{GHz}$ ), NIR/optical (NTT) and $\mathrm{X}$-ray/soft $\gamma$-ray data (PCA, HEXTE and ISGRI instruments from 3 up to $200 \mathrm{keV}$ ) during the second INTEGRAL ToO (Rev. 349). A simple power law is plotted through the radio data to show the optical excess: at least 3 distinct contributions (jet, disc, hot medium) are needed to fit the data.

$10^{30}-10^{33} \mathrm{erg} \mathrm{s}^{-1}$ in, e.g., XTE J1550-564 and H 1743-322: Corbel et al. 2006, and references therein); later, the source did eventually return to quiescence. Several studies on BH LMXBs have shown that such sources can spend weeks to months or even years in a given spectral state (e.g., XTE J1720-318, Cadolle Bel et al. 2004; XTE J650-500, Corbel et al. 2004, SWIFT J1753.5-0127, Soleri et al. 2008). The spectral evolution of XTE J1818-245 is therefore quite typical when compared to other BH LMXBs.

\subsection{Spectral Energy Distribution (SED)}

Two SEDs composed of simultaneous radio, optical, NIR and $\mathrm{X}$-ray/soft $\gamma$-ray data are shown in Figs. 10 and 11. In Fig. 10, we plot the data obtained during the first INTEGRAL ToO (Rev. 348): strong variation in the VLA flux at $4.9 \mathrm{GHz}$ was observed, with the flux increasing by $\sim 20 \mathrm{mJy}$ in less than one day. For the second SED (Rev. 349, Fig. 11), three radio data points are shown at $1.4,4.9$ and $8.4 \mathrm{GHz}$ (the $15 \mathrm{GHz}$ upper limit was omitted for clarity) and we added the NTT magnitudes corrected for absorption (as described in Sect. 3.5.1).

Firstly, when fitting separately the radio and the NIR/optical data with a simple power law, we obtain clearly distinct slopes. From the SED obtained with the most complete coverage (Rev. 349, Fig. 11), we derived a radio spectral index $\alpha$ (where $S_{v} \propto v^{\alpha}$ ) close to 0 for the (non-strictly simultaneous) multifrequency radio data, while the NIR/optical spectral index was +1.0 . In the SED plot the spectral photon indexes $\Gamma$ (where $v F_{v} \propto v^{-\Gamma}$ ) were thus close to -1 and -2 , respectively. Extending the power law spectrum derived from the radio data points up to the NIR/optical frequencies with the same slope, we inferred that the radio (whether from a jet or flaring events) could contribute significantly to the NIR data. However, emission in excess to that extrapolation was also seen in the optical bands, as suggested by the simple power law plotted through the radio data in Fig. 11. This emission probably originates in both the disc and the companion star. Finally, a break was necessary to account for the X-ray/soft $\gamma$-ray data, well fitted by a steeper slope than in 
radio/NIR/optical, with a very soft power law photon index value $(+2.34)$ : the hot medium was thus detected. Therefore at least three components were needed to fit our broad band spectra, from radio to $\mathrm{X} / \gamma$-rays, probably accounting for the presence of matter from a previous ejection event, a disc (plus the companion star) and a hot medium. However, we did not have enough data in the required range $(0.1-2 \mathrm{keV}$ and in the optical) to precisely constrain the SEDs: the disc contribution was too small.

The shape of the SED is similar to the one of the transient LMXB XTE J1118+480 (Chaty et al. 2003; Zurita et al. 2006): although in the LHS during its 2005 outburst, the latter work gave IR and optical slopes with different $\alpha$ values, softening from 0.49 at the peak, not far from our value but distinct, as XTE J1818-245 was in another spectral state, to 0.25 (decay). In the case of the 2000 outburst, the SED of XTE J1118+480 from radio to $\mathrm{X}$-rays has been explained as a combination of synchrotron radiation from a jet and a truncated optically thick disc, whereas models assuming advection dominated accretion flows alone underestimated the optical and IR fluxes (Zurita et al. 2006 and references therein). In 2005, discrepancies observed between the optical and IR SEDs suggested that the IR was dominated by a different component (a jet?) whereas in the optical, the authors were targeting the disc evolution (as we show in this work for XTE J1818-245). Linear fits to optical SEDs have also been performed for other BH XNe in outburst (Hynes 2005): results are relatively uniform and all SEDs exhibit quasi power law spectra, with $\alpha$ ranging from $0.5-1.5$ (compatible with our spectral index of 1), all steeper than the canonical $S_{v} \propto v^{1 / 3}$. In particular, Hynes et al. (2002) observed XTE J1859+226 during the decline from its 1999-2000 outburst: in the soft state, the slopes derived were always between 0.1 and 0.5 . This is slightly different to our value found in the SIMS: we took it closer to the peak. The authors found that the UV/optical/X-ray data - when detectable - could be fitted with a simple black-body model of an accretion disc heated by internal viscosity and X-ray irradiation, but the inner radius could not be well constrained. They concluded that the flat-spectrum synchrotron emission may be important in the IR and optical in this source. However, they did not exclude the alternative explanation that the IR excess could come from the cool outer disc. In our case, although we do not have enough broad-band and time coverage with our data to constrain models as in the previous works, the contribution of the radio (from a jet or flares, see Sect. 4.3) to synchrotron emission up to the NIR/optical could be important, but another component (for example from the cooling disc and/or the companion, or even an irradiated disc, see Sects. 4.1 and 4.2) is necessary to account for the NIR/optical excess observed.

\subsection{Radio constraints and links with $X / \gamma$-rays}

The VLA radio observations of XTE J1818-245 started around 6 days after the ASM peak of the X-ray outburst. While the presence of a possible major flare associated with this outburst was not covered by these data, two radio flares were detected 7 and 15 days after the soft X-ray peak. The first one occurred around MJD 53603 when the source was still in the SIMS; no spectral information is available for this flare. A few days later, when the source was in the harder part of the SIMS, it displayed the behaviour shown in Fig. 9, reminiscent of the radio flares displayed by GRS $1915+105$ (Mirabel et al. 1998). The lack of strictly simultaneous multi-frequency light curves prevents us from deriving the spectral behaviour of the source.

The VLBA upper limits of $1 \mathrm{mJy}^{\text {beam }}{ }^{-1}$ at both 2.3 and $8.4 \mathrm{GHz}$ were obtained with data taken during the second hour of this VLA run and lasting two more hours. These upper limits could indicate either that the source has faded significantly, as suggested by the VLA $15 \mathrm{GHz}$ upper limit, or that the source has slightly expanded, lowering the flux of compact emission with respect to the total emission seen at VLA scales. The lack of simultaneous VLA observations covering the whole VLBA run prevents us from reaching a firm conclusion in this respect. The second radio flare took place around MJD 53611, just before a transition to the HSS (see HID in Fig. 4). The radio spectral index $(\alpha=-0.8 \pm 0.1)$ obtained during this observation is typical of optically thin synchrotron radiation, probably as a result of freely expanding plasma blobs previously ejected (see, e.g., Fender et al. 2004). This suggests that multiple ejection events took place in the outburst of XTE J1818-245. Interestingly, the source was also detected on MJD 53617 , five days after the start of the HSS. The spectral index is very negative, suggesting that this might be residual emission from the second flare. However, the large error bars due to the faintness of the source prevent us from being sure about this result.

\section{Summary and conclusions}

During its 2005 outburst, XTE J1818-245 showed light curves and spectral evolution of a LMXB in outburst, probably with a $\mathrm{BH}$ as the compact object. Spectral parameters were typical of soft-intermediate and high-soft states, showing the usual decrease in disc temperature, increase in inner disc radius, and the decreasing disc flux as the high-energy flux becomes comparatively stronger. However, the track in the HID was not completely standard, and it was accompanied by at least two radio flares in the soft intermediate state, without strong hard X-ray changes. Even if there was an evolution in the power law index and flux, we did not observe the source switching to the LHS as we did for XTE J1720-318 (Cadolle Bel et al. 2004) or XTE J1817-330 (Cadolle Bel et al. 2008).

Radio flares have now been seen in a large number of sources while transiting from HIMS to SIMS (see, e.g., Fender et al. 2004). Several models attempt to characterize the expelled material, the simplest of which is adiabatically expanding material ejected by the system, detectable from its synchrotron emission (van der Laan 1966). In this model, a lag of several tens of minutes is expected between the high and low radio radio frequencies. Such a delay has now been seen in several sources (see, e.g., Mirabel et al. 1998; Fender et al. 2004; Rodriguez et al. 2008a,b). In the case of XTE J1818-245, the two radio flares were clearly associated with two different ejection events. The fact we detected radio emission 5 days after the source entered the HSS is intriguing and cannot be due to the residuals of the flare. One could compare this behaviour to XTE J1650-500 or XTE J1550-564 (Corbel et al. 2004), where the authors surprisingly detected a faint level of radio emission in the soft state. It was consistent with the emission of previously ejected material interacting with the interstellar medium: this might also be the case here.

In most $\mathrm{BH}$ binaries, a major ejection event occurs when the compact jet, present in the LHS in the very first stage of an outburst, turns off as the source enters the HSS. But in some cases the source then alternates between softer and harder states, and repeated ejections on a timescale of days to months may occur. This was the case for, e.g., A0620-00, GRO J1655-40 and XTE J1859+226 (Harmon et al. 1995; Kuulkers et al. 1999; Brocksopp et al. 2002). However, contrary to what was observed in these sources, we did not observe a clear correlation between the (hard) X-ray spectral behaviour and the occurrences of the 
radio flares in XTE J1818-245. Although some "switches" back and forth between SIMS and HSS were seen in the HID, this source remained in rather soft states, and only the last radio flare event was followed by a spectral state transition to a HSS, as in XTE J1859+226 (Brocksopp et al. 2002).

Our analysis clearly shows the importance of a simultaneous multiwavelength and monitoring campaign of sources in outburst. In particular, and although XTE J1818-245 shows quite common behaviour for a $\mathrm{BH}$ in outburst, some specific points are quite intriguing. Constraints from the modeling of the SEDs revealed that its behaviour was not fully compatible with the interpretation formed for other sources in terms of radio/X-ray evolution. The radio extrapolation might be interpreted as non-thermal emission from ejected material, such as discrete ejection events that rapidly faded, except for residual emission seen a few days after entering the HSS. The radio, whether emitted by a jet or as flaring events, could contribute significantly to the NIR data, but an excess was seen and could be explained by contributions from the cooling disc plus the companion. However, alternative processes, such as X-ray irradiation, are sometimes inferred, depending on the source and data available, but there is no consensus yet on that issue (even for the same source), nor on the exact disc geometry/hard radiation origin. Clearly, the diversity and complexity of the interplay of the different emitting media during state transitions need more campaigns and observations to be better understood.

Acknowledgements. We thank the referee for help with our manuscript. We thank the INTEGRAL, Swift and RXTE mission planners for programming the ToO observations described in the paper. L. B. acknowledges support from the Faculty of the European Space Astronomy Center (ESAC). The authors thank M. Nowak for his help in the SED, P. Kretschmar and S. Migliari for their general advice. M.R. and J.M. acknowledge support by DGI of the Spanish Ministerio de Educación y Ciencia (MEC) under grant AYA2007-68034-C03-01 and FEDER funds. M.R. acknowledges financial support from MEC and European Social Funds through a Ramón y Cajal fellowship. DCH is grateful to the Academy of Finland for a Fellowship project number 212656. The present work is based on observations with INTEGRAL, an ESA project with instruments and science data center funded by ESA member states (especially the PI countries: Denmark, France, Germany, Italy, Switzerland, Spain, Czech Republic and Poland, and with the participation of Russia and the USA), and with RXTE. The NRAO is a facility of the National Science Foundation operated under cooperative agreement by Associated Universities, Inc. This research has made use of the NASA Astrophysics Data System Abstract Service and of the SIMBAD database, operated at the CDS, Strasbourg, France.

\section{References}

Arnaud, K. A. 1996, ASP Conf., 101, 17

Belloni, T. 2005, AIP Conf., 797, 197

Belloni, T., Méndez, M., \& Sánchez-Fernández, C. 2001, A\&A, 372, 551

Belloni, T., Homan, J., Casella, P., et al. 2005, A\&A, 440, 207

Bertin, E., \& Arnouts, S. 1996, A\&AS, 117, 393

Bohlin, R. C., Savage, B. D., \& Drake, J. F. 1978, ApJ, 224, 132

Brocksopp, C., Jonker, P. G., Fender, R. P., et al. 2001, MNRAS, 323, 517

Brocksopp, C., Fender, R. P., McCollough, M., et al. 2002, MNRAS, 331, 765

Cadolle Bel, M., Rodriguez, J., Sizun, P., et al. 2004, A\&A, 426, 659

Cadolle Bel, M., Sizun, P., Goldwurm, A., et al. 2006, A\&A, 446, 591

Cadolle Bel, M., Ribó, M., Rodriguez, J., et al. 2007, ApJ, 659, 549

Cadolle Bel, M., Kuulkers, E., Barragán, L., et al. 2008, Proceedings of A Population Explosion (St Petersburg/FL, USA), 28 Oct.-02 Nov. 2007
Casella, P., Belloni, T., \& Stella, L. 2005, ApJ, 629, 403

Chaty, S., Haswell, C. A., Malzac, J., et al. 2003, MNRAS, 346, 689

Chen, W., Shrader, C. R., \& Livio, M. 1997, ApJ, 491, 312

Chincarini, G., Zerbi, F., Antonelli, A., et al. 2003, The Messenger, 113, 40

Coppi, P. S. 1999, ASP Conferences Series, 161, 375

Corbel, S., Fender, R. P., Tzioumis, A. K., et al. 2000, A\&A, 359, 251

Corbel, S., Nowak, M. A., Fender, R. P., Tzioumis, A. K., \& Markoff, S. 2003, A\&A, 400, 1007

Corbel, S., Fender, R. P., Tomsick, J. A., Tzioumis, A. K., \& Tingay, S. 2004, ApJ, 617, 1272

Corbel, S., Tomsick, J. A., \& Kaaret, P. 2006, ApJ, 636, 971

Covino, S., Stefanon, M., Sciuto, G., et al. 2004, Proc. SPIE, 5492, 1613

Done, C., Gierliński, M., \& Kubota, A. 2007, A\&A Rev., 15, 1

Fender, R., Corbel, S., Tzioumis, T., et al. 1999, ApJ, 519, L165

Fender, R. P., Belloni, T. M., \& Gallo, E. 2004, MNRAS, 355, 1105

Fender, R., Belloni, T., \& Gallo, E. 2005, Ap\&SS, 300, 1

Fitzpatrick, E. L. 1999, PASP, 111, 63

Gallo, E., Fender, R. P., \& Pooley, G. G. 2003, MNRAS, 344, 60

Gallo, E., Fender, R. P., Miller-Jones, J. C. A., et al. 2006, MNRAS, 370, 1351

Gierliński, M., Done, C., \& Page, K. 2008, MNRAS, 388, 753

Goldwurm, A., David, P., Foschini, L., et al. 2003, A\&A, 411, L223

Gros, A., Goldwurm, A., Cadolle-Bel, M., et al. 2003, A\&A, 411, L179

Harmon, B. A., Wilson, C. A., Zhang, S. N., et al. 1995, Nature, 374, 703

Homan, J., \& Belloni, T. 2005, Ap\&SS, 300, 107

Homan, J., Wijnands, R., van der Klis, M., et al. 2001, ApJS, 132, 377

Hynes, R. I. 2005, ApJ, 623, 1026

Hynes, R. I., Haswell, C. A., Chaty, S., Shrader, C. R., \& Cui, W. 2002, MNRAS, 331,169

Jahoda, K., Markwardt, C. B., Radeva, Y., et al. 2006, ApJS, 163, 401

King, A. R., \& Ritter, H. 1998, MNRAS, 293, L42

Kuulkers, E., Fender, R. P., Spencer, R. E., Davis, R. J., \& Morison, I. 1999, MNRAS, 306, 919

Kuulkers, E., Shaw, S. E., Paizis, A., et al. 2007, A\&A, 466, 595

Levine, A. M., Swank, J. H., Lin, D., \& Remillard, R. A. 2005, Atel, 578

Markwardt, C. B., Strohmayer, T. E., Swank, J. H., \& Levine, A. M. 2005, Atel, 579

McClintock, J. E., \& Remillard, R. A. 2006, Black hole binaries, Compact stellar X-ray sources, ed. W. Lewin, \& M. van der Klis (Cambridge University Press), 157

Miller, J. M., Homan, J., Steeghs, D., et al. 2006, ApJ, 653, 525

Mirabel, I. F., Dhawan, V., Chaty, S., et al. 1998, A\&A, 330, L9

Poutanen, J., \& Svensson, R. 1996, ApJ, 470, 249

Rodriguez, J., Corbel, S., \& Tomsick, J. A. 2003, ApJ, 595, 1032

Rodriguez, J., Hannikainen, D. C., Shaw, S. E., et al. 2008a, ApJ, 675, 1436

Rodriguez, J., Shaw, S. E., Hannikainen, D. C., et al. 2008b, ApJ, 675, 1449

Rupen, M. P., Dhawan, V., \& Mioduszewski, A. J. 2005, Atel, 589

Rykoff, E. S., Miller, J. M., Steeghs, D., \& Torres, M. A. P. 2007, ApJ, 666, 1129

Schlegel, D. J., Finkbeiner, D. P., \& Davis, M. 1998, ApJ, 500, 525

Shahbaz, T., Charles, P. A., \& King, A. R. 1998, MNRAS, 301, 382

Shaw, S. E., Kuulkers, E., Turler, M., et al. 2005, Atel, 583

Soleri, P., Altamirano, D., Fender, R., et al. 2008, A population explosion, AIPC, 1010, 103

Steeghs, D., Torres, M. A. P., Pych, W., \& Thompson, I. 2005, Atel, 585

Still, M., Gehrels, N., Steeghs, D., et al. 2005, Atel, 588

Sunyaev, R. A., \& Titarchuk, L. G. 1980, A\&A, 86, 121

Tanaka, Y., \& Lewin, W. H. G. 1995, in X-ray Binaries, ed. Lewin, van Paradijs, \& van den Heuvel (Cambridge University Press), 126

Tanaka, Y., \& Shibazaki, N. 1996, ARA\&A, 34, 607

Titarchuk, L. 1994, ApJ, 434, 570

van der Klis, M. 2006, in Compact Stellar X-ray Sources, ed. W. Lewin \& M. van der Klis (Cambridge University Press), 39

van der Laan, H. 1966, Nature, 211, 1131

Westergaard, N. J., Kretschmar, P., Oxborrow, C. A., et al. 2003, A\&A, 411, L257

Zerbi, R. M., Chincarini, G., Ghisellini, G., et al. 2001, AN, 322, 275

Zimmerman, E. R., Narayan, R., McClintock, J. E., \& Miller, J. M. 2005, ApJ, 618, 832

Zurita, C., Torres, M. A. P., Steeghs, D., et al. 2006, ApJ, 644, 432 